\title{
Bounding the First Excursion Probability of Linear Structures Subjected to Imprecise Stochastic Loading
}

\author{
Matthias Faes ${ }^{\mathrm{a}, *}$, Marcos A. Valdebenito ${ }^{\mathrm{b}}$, David Moens ${ }^{\mathrm{a}}$, Michael Beer ${ }^{\mathrm{c}, \mathrm{d}, \mathrm{e}}$ \\ ${ }^{a}$ KU Leuven, Department of Mechanical Engineering, Jan De Nayerlaan 5, St.-Katelijne-Waver, Belgium \\ ${ }^{b}$ Universidad Tecnica Federico Santa Maria, Dept. de Obras Civiles, Av. España 1680, Valparaiso, Chile \\ ${ }^{c}$ Institute for Risk and Reliability, Leibniz Universität Hannover, Callinstr. 34, 30167 Hannover, Germany \\ ${ }^{d}$ Institute for Risk and Uncertainty and School of Engineering, University of Liverpool, Peach Street, Liverpool L69 \\ $7 Z F, U K$ \\ ${ }^{e}$ International Joint Research Center for Engineering Reliability and Stochastic Mechanics, Tongji University, 1239 \\ Siping Road, Shanghai 200092, P.R. China
}

\section{Abstract}

This paper presents a highly efficient and accurate approach to determine the bounds on the first excursion probability of a linear structure that is subjected to an imprecise stochastic load. Traditionally, determining these bounds involves solving a double loop problem, where the aleatory uncertainty has to be fully propagated for each realization of the epistemic uncertainty or vice versa. When considering realistic structures such as buildings, whose numerical models often contain thousands of degrees of freedom, such approach becomes quickly computationally intractable. In this paper, we introduce an approach to decouple this propagation by applying operator norm theory. In practice, the method determines those epistemic parameter values that yield the bounds on the probability of failure, given the epistemic uncertainty. The probability of failure, conditional on those epistemic parameters, is 20 then computed using the recently introduced framework of Directional Importance Sampling. Two case ${ }_{21}$ studies involving a modulated Clough-Penzien spectrum are included to illustrate the efficiency and 22 exactness of the proposed approach.

Keywords: Stochastic loading, First excursion probability, Linear structure, imprecise probabilities, ${ }_{24}^{24}$ interval analysis

Highlights:

- Effects of imprecision on stochastic loads are considered.

- Reliability of linear dynamical structures is quantified.

- Propagation of epistemic and aleatory uncertainty is decoupled.

- Epistemic uncertainty is propagated using operator norm theorem. 
- Probabilities are computed by means of directional importance sampling.

\section{Introduction}

Dynamic loading acting on structural systems can be seldom described precisely. A classical means 33 for characterizing uncertainty in loading and capturing time correlations is resorting to probability 34 theory and in particular, to stochastic processes, see, e.g. [1, 2]. The framework associated with 35 stochastic processes provides an excellent means for capturing inherent (aleatory) uncertainty. However, 36 issues such as lack of knowledge, conflicting sources of information, vagueness and other epistemic 37 sources of uncertainty may hinder the application of stochastic processes. In such context, imprecise 38 probability (see, e.g. [3]) may offer an appropriate framework for handling both types of uncertainties. 39 While imprecise probability is a versatile tool, it also poses a major challenge from a numerical viewpoint 40 when performing uncertainty quantification, as both sources of uncertainty (aleatory and epistemic) 41 must be propagated to the response of the structural system. In view of such issue, this contribution 42 proposes an approach for dealing with both sources of uncertainties by means of a decoupling strategy, 43 which allows a drastic reduction on numerical efforts when compared to existing alternatives in the 44 literature (see, e.g. [3]). Decoupling is investigated herein for the specific case of the estimation of 45 interval first excursion probability, that quantifies the level of safety of a structure under dynamic 46 loading.

Imprecise probabilistic analysis as described above [4] offers a variety of tools to deal with such 48 "deep" (i.e., a combination of aleatory and epistemic) uncertainty. Following for instance a p-box 49 framework, the epistemically uncertain hyper-parameters of the random parameter distributions are 50 modeled as being interval or fuzzy valued [4], and propagated as such through the numerical simulation 51 model under consideration. It is important to note that such propagation is conducted under the 52 condition that the effects of aleatory and epistemic uncertainty are kept separated. This implies that 53 both sources of uncertainty are usually propagated by means of the so-called double loop approaches, 54 where the outer loop takes care of epistemic uncertainty while the inner loop deals with aleatory 55 uncertainty [5]. Double loop approaches such as e.g., applied in [6] are generally highly accurate, but 56 the corresponding computational cost becomes quickly intractable, especially when industrially sized 57 models are considered. Therefore, a considerable amount of research is focused on finding more efficient 58 techniques for the propagation of deep uncertainty through numerical simulation models. In this context, 59 several authors proposed approaches that rely on the approximation of the interval valued parameters ${ }_{60}$ 
via series expansion methods (see e.g., [7], [8]) or orthogonal polynomial expansion schemes (see e.g., 61 [9]), effectively enabling propagation without double loop approaches. However, in case the epistemic 62 uncertainty is comparatively large, perturbation approaches are known to be inaccurate [10], a problem ${ }^{63}$ that is alleviated by resorting to Chebyshev polynomial based schemes such as presented in [11]. Also ${ }^{64}$ efficient surrogate modeling schemes for imprecise probabilistic problems have been proposed using 65 sparse polynomial chaos expansion representations of the model (see e.g., $[12,13]$ ), interval predictor ${ }_{6}$ models $[14,15]$ or variants of the Sobol-Hoeffding decomposition (also known as HDMR representation) ${ }^{67}$ of the relation between the epistemic parameters and the probability of failure [16, 17], providing an 68 efficient and accurate approximation of the problem. Yet another type of methods for propagating 69 mixed uncertainty rely on extensions of classical methods for structural reliability, see e.g. [18, 19]. 70 In the context of imprecise probabilistic stochastic processes, such as the ones described in the first 71 paragraph, only very recent initiatives have been undertaken. Gao et al. [20] introduced imprecise 72 random fields where the mean and variance of the field are interval valued. Dannert et al. [21] and 73 Faes and Moens [22] introduced random fields where also the correlation length of the auto-correlation 74 function can be interval valued. Alternative approaches to deal with sources of insufficient data in 75 the description of quantities that are subjected to uncertainty with spatial correlation include methods 76 based on (Bayesian) compressive sampling [23, 24] or Kriging regression models [25].

This paper deals with bounding the first excursion probability of linear systems subjected to an 78 imprecise stochastic excitation. By fully exploiting the linearity in this problem, the method that is 79 introduced in this paper efficiently and effectively computes these bounds by applying operator norm 80 theory. Specifically, operator norms are applied to find those epistemic parameters that yield a bound ${ }_{81}$ on the probability of failure a priori, requiring only a single deterministic model evaluation together 82 with the solution of two optimization problems. Then, based on the identified values for the epistemic 83 uncertain parameters, the bounds on the first excursion probability can be obtained by propagating two 84 stochastic processes through the numerical model, which is sped up by applying Directional Importance 85 Sampling [26]. In this paper, the application of the method is examined on the computation of the first 86 excursion probability of a structure that is subjected to a stochastic ground acceleration, which in its 87 turn is modeled as a modulated Clough-Penzien spectrum (see e.g. [1, 2]). A first case study illustrates 88 the method on a single-degree-of-freedom (SDOF) oscillator. This study shows that the method is more 89 efficient than double loop approaches where quasi Monte Carlo or the vertex approach are used for the 90 propagation of the epistemic uncertainty. Furthermore, it is shown that the proposed approach is also 91 
far more accurate than both approaches. The second case study concerns a finite element model of a 92 6-story building subjected to a modulated Clough-Penzien spectrum. Also in this case, the accuracy 93 and efficiency of the method is illustrated. This paper is structured as follows. Section 2 provides a 94 rigorous formulation of the problem under consideration. Section 3 presents directional Importance 95 Sampling as an extension to the directional sampling method. Section 4 discusses the computation of 96 the reliability of structures subjected to imprecise stochastic loading, both via a traditional double-loop 97 approach, as well as via the presented decoupling approach. Section 5 and 6 provide the illustrative 98 case studies. Finally, Section 7 lists the conclusions of this manuscript.

\section{Formulation of the Problem}

This section describes the class of problems considered in this contribution, namely calculation of 101

the bounds of first excursion probabilities of linear systems subjected to imprecise stochastic ground 102 acceleration loads. The presented material starts with a formulation considering purely aleatory uncer- 103 tainty and then, the effects of imprecision are included. Such flow of ideas is selected as the problem of 104 calculating first excursion probabilities in the presence of aleatory uncertainty is already quite involved; 105 thus the consideration of imprecision makes this problem even more challenging.

\subsection{Dynamic Analysis}

Consider a structural system modeled as linear, elastic and with classical damping. The model 108 possesses $n_{D}$ degrees-of-freedom, its structural matrices are deterministic, and it is subjected to a 109 stochastic loading $p(t)$. The equation of motion is [27]:

$$
\boldsymbol{M} \ddot{\boldsymbol{x}}(t, \boldsymbol{z})+\boldsymbol{C} \dot{\boldsymbol{x}}(t, \boldsymbol{z})+\boldsymbol{K} \boldsymbol{x}(t, \boldsymbol{z})=\boldsymbol{\rho} p(t, \boldsymbol{z}), t \in[0, T], \boldsymbol{x}(0, \boldsymbol{z})=\dot{\boldsymbol{x}}(t, \boldsymbol{z})=\mathbf{0}
$$

where $\boldsymbol{x}, \dot{\boldsymbol{x}}$ and $\ddot{\boldsymbol{x}}$ are vectors that represent the displacement, velocity and acceleration, respectively, 111 each of dimension $n_{D} \times 1 ; \boldsymbol{M}, \boldsymbol{C}$, and $\boldsymbol{K}$ are the mass, damping and stiffness matrices, respectively, 112 each of dimension $n_{D} \times n_{D}$. Vector $\boldsymbol{\rho}$ couples the stochastic loading $p(t)$ with the corresponding 113 degrees-of-freedom of the structure and its dimension is $n_{D} \times 1$.

In case where the effects of ground accelerations on a structure are considered, for instance to study 115 the loads induced by earthquakes, the inherent uncertainty associated with this ground acceleration can 116 be described in terms of a stochastic Gaussian processes $P(t)[28,29,30,31]$. Hereto, let $p(t)$ in Eq. (1) 117 denote a Gaussian ground acceleration acting over a structural system that is dependent on time $t$. 118 
Without loss of generality, it is assumed in the following that the mean value of this process is zero. In 119 a first approximation, $P(t)$ is regarded as a wide-sense stationary process which can be characterized 120 through its power spectral density $S(\omega)$, where $\omega$ denotes circular frequency. The Wiener-Khintchine 121 theorem (see, e.g. [32]) allows for the calculation of the autocorrelation function of a stochastic process 122 from its power spectrum and vice versa based on following Fourier transforms:

$$
\begin{aligned}
& S(\omega)=\frac{1}{2 \pi} \int_{-\infty}^{+\infty} R(\tau) e^{-i \omega \tau} d \tau \\
& R(\tau)=\int_{-\infty}^{+\infty} S(\omega) e^{i \omega \tau} d \omega
\end{aligned}
$$

with $R(\tau)$ the autocorrelation function with lag $\tau$.

The above discussion assumes that the stochastic process can be modeled as a wide-sense stationary 125 stochastic process. It is clear that this is a simplifying assumption, as loading may in realistic conditions 126 exhibit a non stationary behavior. A possible means for including such effect consists of modulating the ${ }_{127}$ stationary stochastic process by means of a deterministic function of time $m(t)$ (see, e.g. [33]). Thus, 128 the autocorrelation function of the stochastic process $R^{\mathrm{m}}$ becomes [34]:

$$
R^{\mathrm{m}}\left(t_{1}, t_{2}\right)=m\left(t_{1}\right) m\left(t_{2}\right) R^{\mathrm{s}}\left(t_{2}-t_{1}\right)
$$

where $t_{1}$ and $t_{2}$ are two time instants and $R^{s}$ is the stationary autocorrelation function of the stochastic $\quad 130$ process, before being modulated.

Samples of the stochastic process as described above can be generated applying the Karhunen-Loève 132 (KL) expansion (see, e.g. $[35,36]$ ). For this purpose, assume that the loading possesses a duration $T$ and 133 that time is discretized such that $t_{k}=(k-1) \Delta t, k=1, \ldots, n_{T}$, where $\Delta t$ is the time step discretization 134 and $n_{T}$ the number of discrete time steps. Then, the discrete covariance matrix $\boldsymbol{\Gamma}$ of dimension $n_{T} \times n_{T} \quad{ }_{135}$ associated with the stochastic loading model becomes:

$$
\boldsymbol{\Gamma}=\left[\begin{array}{cccc}
m\left(t_{1}\right) m\left(t_{1}\right) R^{\mathrm{s}}(0) & m\left(t_{1}\right) m\left(t_{2}\right) R^{\mathrm{s}}\left(t_{1}-t_{2}\right) & \ldots & m\left(t_{1}\right) m\left(t_{n_{T}}\right) R^{\mathrm{s}}\left(t_{1}-t_{n_{T}}\right) \\
m\left(t_{2}\right) m\left(t_{1}\right) R^{\mathrm{s}}\left(t_{2}-t_{1}\right) & m\left(t_{2}\right) m\left(t_{2}\right) R^{\mathrm{s}}(0) & \ldots & m\left(t_{2}\right) m\left(t_{n_{T}}\right) R^{\mathrm{s}}\left(t_{2}-t_{n_{T}}\right) \\
\vdots & \vdots & \ddots & \vdots \\
m\left(t_{n_{T}}\right) m\left(t_{1}\right) R^{\mathrm{s}}\left(t_{n_{T}}-t_{1}\right) & m\left(t_{n_{T}}\right) m\left(t_{2}\right) R^{\mathrm{s}}\left(t_{n_{T}}-t_{2}\right) & \ldots & m\left(t_{n_{T}}\right) m\left(t_{n_{T}}\right) R^{\mathrm{s}}(0)
\end{array}\right]
$$

Samples of the stochastic ground acceleration can be generated according to the well-known Karhunen- ${ }_{137}$ 
Loève expansion:

$$
\boldsymbol{p}(\boldsymbol{z})=\boldsymbol{\Psi} \Lambda^{1 / 2} \boldsymbol{z}
$$

where $\boldsymbol{p}$ denotes a $n_{T} \times 1$ vector containing the sample of the loading; $\boldsymbol{z}$ is a realization of the random 139 variable vector $\boldsymbol{Z}$ which follows a $n_{K L}$-dimensional standard Gaussian distribution; $n_{K L}$ is the number ${ }_{140}$ of terms retained in the KL expansion; $\boldsymbol{\Psi}$ is a $n_{T} \times n_{K L}$ matrix whose columns contain the eigenvectors 141 associated with the largest $n_{K L}$ eigenvalues of the discrete covariance matrix $\boldsymbol{\Gamma}$; and $\boldsymbol{\Lambda}$ is a $n_{K L} \times n_{K L} \quad{ }_{142}$ matrix whose diagonal contains the largest $n_{K L}$ eigenvalues of $\boldsymbol{\Gamma}$. A criterion for selecting the number ${ }_{143}$ of terms to be retained in the KL expansion is to find the minimum value of $n_{K L}$ such that $\sum_{p=1}^{n_{K L}} \lambda_{p} \geq{ }_{144}$ $p_{v} \sum_{p=1}^{n_{T}} \lambda_{p}$, where $p_{v}$ denotes the fraction of the total variance of the underlying stochastic process that ${ }_{145}$ is retained by the approximate representation and $\lambda_{p}$ is the $p$-th eigenvalue of $\boldsymbol{\Gamma}$ [37]. For a recent ${ }_{146}$ overview of numerical methods to solve the associated Fredholm integral eigenvalue problem, the reader ${ }_{147}$ is kindly referred to the overview paper by Betz et al. [38]. 148

In general engineering practice, only a subset of all dynamic responses $\boldsymbol{x}$ of the structure in eq. (1) ${ }_{149}$ are of interest for the analysis. These dynamic responses are denoted as $\eta_{i}(t, \boldsymbol{z}), i=1, \ldots, n_{\eta}$ and are 150 calculated applying the convolution integral between the corresponding unit impulse response functions 151 $h_{i}(t), i=1, \ldots, n_{\eta}$ and the stochastic loading $p(t, \boldsymbol{z})$ :

$$
\eta_{i}(t, \boldsymbol{z})=\int_{0}^{t} h_{i}(t-\tau) p(t, \boldsymbol{z}) d \tau, i=1, \ldots, n_{\eta}
$$

Under the assumption that the responses of interest correspond to a linear combination of the response ${ }_{153}$ vector, the unit impulse response functions are calculated as:

$$
h_{i}(t)=\sum_{v=1}^{n_{D}} \frac{\boldsymbol{\gamma}_{i}^{T} \boldsymbol{\phi}_{v} \boldsymbol{\phi}_{v}^{T} \boldsymbol{\rho}}{\boldsymbol{\phi}_{v}^{T} \boldsymbol{M} \boldsymbol{\phi}_{v}} \frac{1}{\omega_{d, v}} e^{-\zeta_{v} \omega_{v} t} \sin \left(\omega_{d, v} t\right), i=1, \ldots, n_{\eta}
$$

where $\phi_{v}, v=1, \ldots, n_{D}$ are the eigenvectors associated with the eigenproblem of the undamped equation of motion; $\omega_{v}, v=1, \ldots, n_{D}$ are the natural frequencies of the system; $\zeta_{v}, v=1, \ldots, n_{D}$ are the corresponding damping ratios; $\omega_{d, v}=\omega_{v} \sqrt{\left(1-\zeta_{v}^{2}\right)}, v=1, \ldots, n_{D}$ are the damped frequencies; and $\gamma_{i}$ is a constant vector such that $\eta_{i}=\boldsymbol{\gamma}_{i}^{T} \boldsymbol{x}$. It should be noted that the contribution of higher order modes to the unit impulse response function in Eq. (7) is negligible for several cases of practical interest 159 $[27]$.

In view of the excitation model introduced in Eq. (5), the dynamic response of interest evaluated at 161 
time $t_{k}$ is:

$$
\begin{aligned}
\eta_{i}\left(t_{k}, \boldsymbol{z}\right) & =\sum_{l_{1}=1}^{k} \Delta t \epsilon_{l_{1}} h_{i}\left(t_{k}-t_{l_{1}}\right)\left(\sum_{l_{2}=1}^{n_{K L}} \psi_{l_{1}, l_{2}} \sqrt{\lambda_{l_{2}}} z_{l_{2}}\right) \\
& =\boldsymbol{a}_{i, k}^{T} \boldsymbol{z}, i=1, \ldots, n_{\eta}, \quad k=1, \ldots, n_{T}
\end{aligned}
$$

where $\psi_{l_{1}, l_{2}}$ is the $\left(l_{1}, l_{2}\right)$-th element of matrix $\boldsymbol{\Psi} ; \boldsymbol{a}_{i, k}$ is a vector of dimension $n_{K L} \times 1$ such that:

$$
\boldsymbol{a}_{i, k}=\left[\begin{array}{c}
\sum_{l_{1}=1}^{k} \Delta t \epsilon_{l_{1}} h_{i}\left(t_{k}-t_{l_{1}}\right) \psi_{l_{1}, 1} \sqrt{\lambda_{1}} \\
\sum_{l_{1}=1}^{k} \Delta t \epsilon_{l_{1}} h_{i}\left(t_{k}-t_{l_{1}}\right) \psi_{l_{1}, 2} \sqrt{\lambda_{2}} \\
\vdots \\
\sum_{l_{1}=1}^{k} \Delta t \epsilon_{l_{1}} h_{i}\left(t_{k}-t_{l_{1}}\right) \psi_{l_{1}, n_{K L}} \sqrt{\lambda_{n_{K L}}}
\end{array}\right]
$$

and $\epsilon_{l_{1}}$ is a coefficient depending on the numerical integration scheme used in the evaluation of the ${ }_{164}$ convolution integral. For the case where the trapezoidal integration rule [39] is chosen, $\epsilon_{l_{1}}=1 / 2$ if ${ }_{165}$ $l_{1}=1$ or $l_{1}=k$; otherwise, $\epsilon_{l_{1}}=1$.

\subsection{Precise reliability analysis}

Structural systems subjected to a stochastic loading as described above exhibit an uncertain re- 168 sponse. A possible means for quantifying such uncertainty consists of calculating the first excursion 169 probability, which measures the probability that any of the responses of interest $\eta_{i}(t), i=1, \ldots, n_{\eta} \quad 170$ exceeds a prescribed threshold level $b_{i}, i=1, \ldots, n_{\eta}$ within the duration $T$ of the stochastic excitation. 171 This failure criterion is cast in terms of the so-called performance function, which is equal to:

$$
g(\boldsymbol{z})=1-\max _{i=1, \ldots, n_{\eta}}\left(\max _{k=1, \ldots, n_{T}}\left(\frac{\left|\eta_{i}\left(t_{k}, \boldsymbol{z}\right)\right|}{b_{i}}\right)\right)
$$

where $|\cdot|$ denotes the absolute value. From this equation, note that the term $\left|\eta_{i}\left(t_{k}, \boldsymbol{z}\right)\right| / b_{i}$ represents the 173 normalized demand, which measures how close the response of interest lies to the allowable threshold in 174 a dimensionless manner [40]. Thus, whenever the maximum normalized demand (retrieved by $\max (\cdot) \quad 175$ in eq. (11)) exceeds unity, failure takes place. The above formulation of the performance function 176 corresponds to a classical series event (see, e.g. [41]). 
As such, the first excursion probability is written in terms of the following integral [32, 41, 42]:

$$
P_{f}=\int_{\boldsymbol{z} \in \mathbb{R}^{n} K L} I_{F}(\boldsymbol{z}) f_{\boldsymbol{Z}}(\boldsymbol{z}) d \boldsymbol{z}
$$

where $f_{\boldsymbol{Z}}(\cdot)$ is standard Gaussian probability density function in $n_{K L}$ dimensions; and $I_{F}(\cdot)$, is an 179 indicator function whose value is equal to one in case $g(\boldsymbol{z})<0$ and zero otherwise. It is noted that 180 the probability integral in Eq. (12) usually comprises a high number of dimensions, as $n_{K L}$ may be in 181 the order of hundreds or thousands, while the indicator function is known point-wise only for specific 182 realizations $\boldsymbol{z}$ of $\boldsymbol{Z}$. Therefore, such an integral cannot be solved analytically; however, lower/upper 183 bounds [43] or approximate solutions [44] exist in certain cases. For more general cases, simulation 184 methods appear to be the only feasible means for evaluating the failure probability, see e.g. [45].

\subsection{Effects of Imprecision on Stochastic Loading and First Excursion Probability}

The characterization of the stochastic process in terms of its power spectral density or autocorrelation 187 function and a modulating function as described above usually relies on a prescribed model, which in 188 its turn depends on a number of parameters, grouped in a vector $\boldsymbol{\theta}$. Without loss of generality, in this 189 paper we consider the modulated Clough-Penzien (CP) autocorrelation function (see also Appendix A). 190 The parameters that determine the auto-correlation function $R^{m}\left(t_{1}, t_{2}\right)$ in this case reflect some specific 191 characteristics of the process, such as dominant frequencies, amplitude, etc. Naturally, when setting the 192 model, there might be considerable uncertainty on the precise values of these parameters [46] arising due 193 to issues such as lack of knowledge, vagueness, conflicting information sources, etc., especially given the 194 fact that these parameters may be highly sensitive to specific site conditions. For instance, considering 195 Table A.7, a selection of the appropriate parameters has to be made based on the classification of the 196 soil being Firm, Medium or Soft. As an illustration of the effect of this imprecision, figure 1 shows 197 the power spectrum corresponding to these three classifications of the soil and a spectral intensity 198 $S_{0}=0.05 \mathrm{~m}^{2} / \mathrm{s}^{3}$. The figure furthermore shows a realization of the stochastic process corresponding to 199 each of these spectra, with parameters of the modulation equal to $c_{1}=0.14, c_{2}=0.16$ (see details in 200 Appendix A), where $\boldsymbol{z}$ is taken to be the same for the three processes. As is clear from this figure, the 201 classification of the soil has a large impact on the representation of the base excitation that is imposed 202 on the structure under consideration.

In such scenario, non-traditional models for uncertainty quantification appear as a natural choice ${ }^{204}$ for characterizing the parameters $\boldsymbol{\theta}$ of the stochastic loading $[4,10]$, as they provide the analyst with 205 

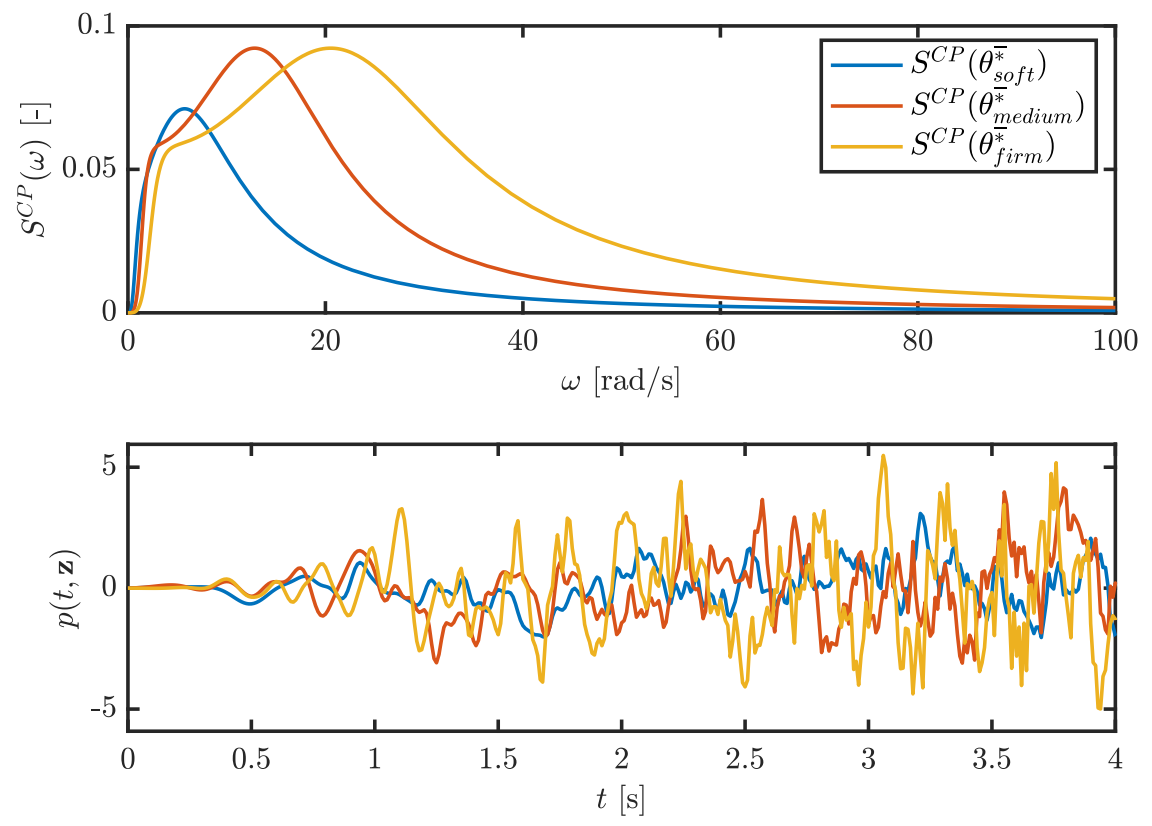

Figure 1: Clough-Penzien spectra corresponding to a soft, medium or firm soil as well as one realisation from the stochastic process corresponding to these spectra.

more objective and robust tools to asses the bounds on the estimated reliability of the structure, based 206 on epistemic uncertainty that is present on the parameters of the stochastic process definition. In 207 this paper, the uncertainty of each of these parameters is characterized as an interval vector $\boldsymbol{\theta}^{I}$, as ${ }_{208}$ intervals require only very few data points to make an objective worst-case estimate of the bounds on 209 the reliability [10]. Furthermore, recent developments allow for estimating robust interval bounds given 210 only limited data (see e.g., $[47,48])$. In the following intervals are denoted with apex I, e.g. $\boldsymbol{\theta}^{I}$ denotes the ${ }_{211}$ interval associated with $\boldsymbol{\theta}$. In the example of the modulated $\mathrm{CP}$ auto-correlation function, $\boldsymbol{\theta}^{I}$ is defined ${ }_{212}$ as $\boldsymbol{\theta}^{I}=\left[\omega_{g}^{I}, \omega_{f}^{I}, \zeta_{g}^{I}, \zeta_{f}^{I}, S_{0}^{I}, c_{1}^{I}, c_{2}^{I}\right]$, which can geometrically be represented as a 7-dimensional hyper- ${ }_{213}$ rectangular input space (please see Appendix A for the physical interpretation of these parameters). $\quad 214$

The fact that the input parameters of the stochastic loading model are described by means of ${ }_{215}$ intervals has important implications on the evaluation of the structural reliability of the model under 216 consideration. In particular, both the loading and the responses of interest of the structural system ${ }_{217}$ become interval stochastic processes, as described in [22]. This implies in turn that the performance ${ }_{218}$ function becomes interval valued, which causes the failure probability to become interval valued as well. 219 This implies that both the lower bound $\underline{P}_{f}$ and upper bound $\bar{P}_{f}$ of the interval associated the failure ${ }_{220}$ 
probability $p_{F}^{I}$ must be determined, which leads to the following two optimization problems:

$$
\begin{gathered}
\underline{P}_{f}=\min _{\boldsymbol{\theta} \in \boldsymbol{\theta}^{I}}\left(P_{f}(\boldsymbol{\theta})\right)=\min _{\boldsymbol{\theta} \in \boldsymbol{\theta}^{I}}\left(\int_{\boldsymbol{z} \in \mathbb{R}^{n} K L} I_{F}(\boldsymbol{z}, \boldsymbol{\theta}) f_{\boldsymbol{Z}}(\boldsymbol{z}) d \boldsymbol{z}\right) \\
\bar{P}_{f}=\max _{\boldsymbol{\theta} \in \boldsymbol{\theta}^{I}}\left(P_{f}(\boldsymbol{\theta})\right)=\max _{\boldsymbol{\theta} \in \boldsymbol{\theta}^{I}}\left(\int_{\boldsymbol{z} \in \mathbb{R}^{n_{K L}}} I_{F}(\boldsymbol{z}, \boldsymbol{\theta}) f_{\boldsymbol{Z}}(\boldsymbol{z}) d \boldsymbol{z}\right)
\end{gathered}
$$

The calculation of the bounds associated with the failure probability can be extremely demanding from 223 a numerical viewpoint. On one hand, the calculation of the failure probability for a fixed value of the 224 parameters associated with the stochastic process is quite costly, even when highly efficient methods 225 such as Directional Importance Sampling (as described in the forthcoming section) are applied. On the 226 other hand, solving the associated optimization problems is far from trivial, as it constitutes a double ${ }^{227}$ loop problem, where the inner loop comprises probability calculation, while the outer loop explores 228

the possible values that the parameters $\boldsymbol{\theta}$ may assume. As such, a full propagation of the stochastic 229 process towards the first excursion probability has to be performed. Hence, apart from considering 230 near-trivial simulation models, such computation is intractable without resorting to surrogate modelling 231 strategies [49]. 232

Such task is carried out as follows in this work: Section 3 addresses the issue of the failure probability ${ }^{233}$ whenever the stochastic loading process is characterized precisely (that is, for a crisp value of $\boldsymbol{\theta}$ ), while ${ }^{234}$ in Section 4, the effect of imprecision on the stochastic loading model is explicitly included in the 235 analysis and the bounds on the interval failure probability are calculated efficiently and accurately with 236 the help of the operator norm theorem.

\section{Directional importance sampling}

\subsection{Context}

This section presents Directional Importance Sampling (DIS), which is a simulation approach that 240 allows calculating first excursion probabilities of linear structural systems subject to Gaussian loading 241 [26]. In the following, it is assumed that the parameters $\boldsymbol{\theta}$ associated with the stochastic loading model 242 can be regarded as deterministic. In this context, note that the estimation of such first excursion 243 probabilities is not trivial (even when precise probabilistic models are considered) and hence, DIS plays 244 a fundamental role for reliability assessment within the proposed framework. 


\subsection{Basic formulation}

Directional sampling (see e.g., [50], [51]) consists of exploring the input space associated with the 247 stochastic loading by random samples of a unit direction vector amplified by a length factor. This 248 corresponds to a polar representation, as discussed in detail in [52]. Thus, the realization $\boldsymbol{z}$ is represented 249 as:

$$
z=r u
$$

where $\boldsymbol{u}$ is a unit vector pointing towards $\boldsymbol{z}$, that is:

$$
\boldsymbol{u}=\frac{\boldsymbol{z}}{\|\boldsymbol{z}\|_{2}}
$$

where $\|\cdot\|_{2}$ denotes the Euclidean norm; and $r$ is the Euclidean norm of $\boldsymbol{z}$, that is $r=\|\boldsymbol{z}\|_{2}$. Thus, the 252 failure probability integral in Eq. (12) is expressed as:

$$
P_{f}=\int_{r} \int_{\boldsymbol{u}} I_{F}(r \boldsymbol{u}) f_{R}(r) f_{\boldsymbol{U}}(\boldsymbol{u}) d \boldsymbol{u} d r
$$

where $f_{\boldsymbol{U}}(\boldsymbol{u})$ is the uniform probability distribution over a hypersphere and $f_{R}(r)$ is the probability 254 density function associated with $r$. It is readily seen that applying the formula for change of variables for 255 probability distributions [53], $f_{R}(r)=2 r f_{\chi_{n_{T}}^{2}}\left(r^{2}\right)$, where $f_{\chi_{n_{T}}^{2}}\left(r^{2}\right)$ is the Chi-squared probability density 256 function of $n_{T}$ degrees-of-freedom (recall that $r^{2}$ follows a Chi-squared distribution of $n_{T}$ degrees-of- 257 freedom as it is the sum of the squares of $n_{T}$ standard Gaussian variables). 258 Direct estimation by means of simulation of Eq. (17) may not be efficient: as failure probabilities are 259 usually low (e.g. $10^{-3}$ or less), the associated estimator may exhibit a large variability. Such issue can 260 be alleviated by introducing a suitable importance sampling density function $f_{\boldsymbol{U}, I S}(\boldsymbol{u})$, which leads to 261 the following expression for the failure probability integral:

$$
P_{f}=\int_{r} \int_{\boldsymbol{u}} I_{F}(r \boldsymbol{u}) f_{R}(r) \frac{f_{\boldsymbol{U}}(\boldsymbol{u})}{f_{\boldsymbol{U}, I S}(\boldsymbol{u})} f_{\boldsymbol{U}, I S}(\boldsymbol{u}) d \boldsymbol{u} d r
$$

The above expression corresponds to a Directional Importance Sampling (DIS) strategy for calculating 263 the failure probability and it can be efficiently estimated by means of random sampling given that the 264 importance sampling density function $f_{\boldsymbol{U}, I S}(\boldsymbol{u})$ is selected appropriately. This issue is discussed in the 265 following. 


\subsubsection{Failure domain and its geometry}

As discussed in [54], the safe and failure domains associated with the first excursion probability of 268 a linear structure subject to Gaussian excitation are separated by a collection of hyperplanes. Such 269 information provides valuable information for selecting $f_{\boldsymbol{U}, I S}(\boldsymbol{u})$.

Consider the so-called positive elementary failure domain $F_{i, k}{ }^{+}$, which is the set that collects all real- ${ }^{271}$ izations $z$ such that the response $\eta_{i}$ exceeds its corresponding threshold $b_{i}$ at the time instant $t_{k}$. In 272 view of linearity of the response of interest with respect to $\boldsymbol{z}$ as shown in Eq. (8), it is noted that the ${ }_{273}$ set $F_{i, k}{ }^{+}$is bounded by a hyperplane, that is:

$$
F_{i, k}^{+}=\left\{\boldsymbol{z} \in \mathbb{R}^{n_{K L}}: \boldsymbol{a}_{i, k}^{T} \boldsymbol{z} \geq b_{i}\right\}
$$

In a similar manner, the negative elementary failure domain $F_{i, k}{ }^{-}$, in which the response of interest $\eta_{i} \quad 275$ exceeds the threshold $-b_{i}$ at the time instant $t_{k}$, is also bounded by a hyperplane:

$$
F_{i, k}^{-}=\left\{\boldsymbol{z} \in \mathbb{R}^{n_{K L}}: \boldsymbol{a}_{i, k}^{T} \boldsymbol{z} \leq-b_{i}\right\}
$$

The union of positive and negative elementary failure domains $F_{i, k}{ }^{+}$and $F_{i, k}{ }^{-}$, respectively, forms the 277 elementary failure domain $F_{i, k}$. In addition, the overall failure event $F$ is formed by the union of 278 elementary failure events (see, e.g. [41]).

$$
F=\bigcup_{i=1}^{n_{\eta}} \bigcup_{k=1}^{n_{T}} F_{i, k}
$$

Note that the failure event $F$ contains all possible random excitations that cause a first excursion. 280 These elementary failure domains are represented schematically in Figure 2.

As the elementary failure events $F_{i, k}$ are bounded by hyperplanes, their individual probability of 282 occurrence is completely characterized by the reliability indexes $\beta_{i, k}, i=1, \ldots, n_{\eta}, k=1, \ldots, n_{T}$. 283 These indexes are equal to $[54,55]$ :

$$
\beta_{i, k}=\frac{b_{i}}{\left\|\boldsymbol{a}_{i, k}\right\|}
$$

Figure 2 provides a schematic representation of the reliability indexes. From this figure, it is easy to see 285 that these indexes are actually equal to the Euclidean norm of the design points associated with each 286 


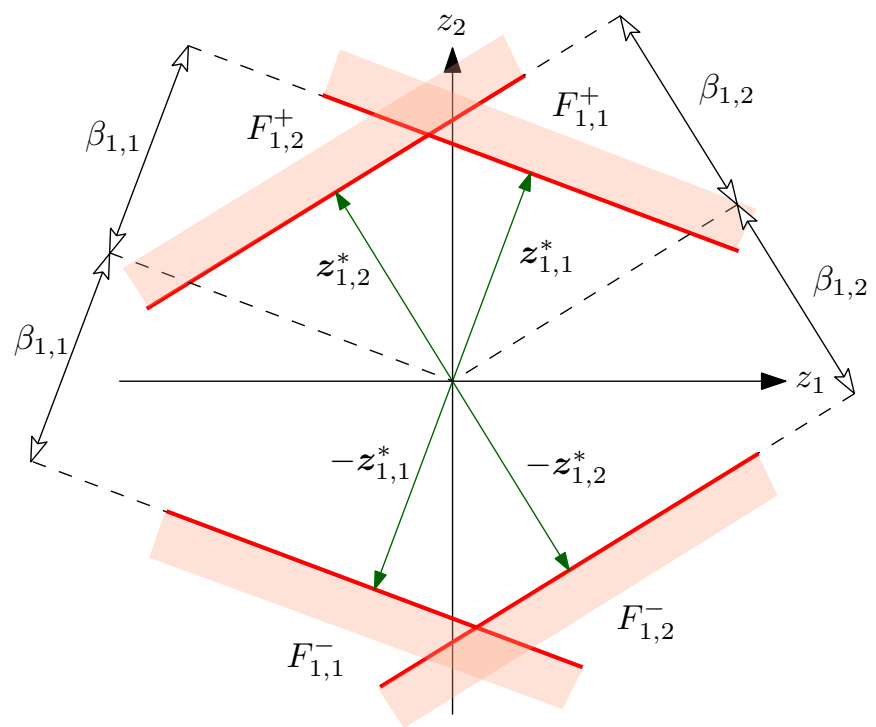

Figure 2: Failure domain for the case where $n_{K L}=2$.

elementary failure domain (please see $[54,55]$ for details on the definition of the design point). Hence, 287 the probability of occurrence of each of the elementary failure events is given by:

$$
P\left[F_{i, k}\right]=2 \Phi\left(-\beta_{i, k}\right)
$$

where $P[\cdot]$ denotes probability and $\Phi(\cdot)$ represents the standard normal cumulative density function. 289 The summation $\hat{P}_{f}$ of the probability of occurrence of each elementary failure event:

$$
\hat{P}_{f}=2 \sum_{i=1}^{n_{\eta}} \sum_{k=1}^{n_{T}} \Phi\left(-\beta_{i, k}\right)
$$

provides an upper bound for the sought first excursion probability [41, 55], that is $P_{f} \leq \hat{P}_{f}$.

As the elementary failure domains are bounded by hyperplanes, it is possible to deduce some analytic 292 expressions associated with them. For example, the probability density associated with a direction $\boldsymbol{u}{ }_{293}$ in the standard normal space given that the elementary failure event $F_{i, k}$ occurs, which is denoted as 294 $f_{\boldsymbol{U}}\left(\boldsymbol{u} \mid F_{i, k}\right)$, can be calculated in closed form by virtue of Bayes' theorem, yielding [26, 52]:

$$
f_{\boldsymbol{U}}\left(\boldsymbol{u} \mid F_{i, k}\right)=\frac{f_{\boldsymbol{U}}(\boldsymbol{u}) P\left[F_{i, k} \mid \boldsymbol{u}\right]}{P\left[F_{i, k}\right]}
$$

The term $P\left[F_{i, k} \mid \boldsymbol{u}\right]$ expresses the probability of occurrence of the elementary failure domain $F_{i, k}$ con- 296 ditioned on the unit direction $\boldsymbol{u}$. It can be readily demonstrated that such probability can be solved in ${ }^{297}$ 
closed form (see detailed deduction in [26]), leading to:

$$
f_{\boldsymbol{U}}\left(\boldsymbol{u} \mid F_{i, k}\right)=\frac{f_{\boldsymbol{U}}(\boldsymbol{u})\left(1-F_{X_{n T}^{2}}\left(C_{i, k}(\boldsymbol{u})^{2}\right)\right)}{P\left[F_{i, k}\right]}
$$

where $F_{\chi_{n T}^{2}}(\cdot)$ corresponds to the Chi-squared cumulative density function with $n_{T}$ degrees-of-freedom 299 and $C_{i, k}(\boldsymbol{u})$ corresponds to the Euclidean norm of the vector lying between the origin of the standard 300 normal space and the intersection of the boundary of $F_{i, k}$ with the ray along the direction $\boldsymbol{u}$. In view 301 of linearity of the response, it is straightforward to demonstrate that $C_{i, k}(\boldsymbol{u})$ is equal to [26]:

$$
C_{i, k}(\boldsymbol{u})=\frac{b_{i}}{\left|\eta_{i}\left(t_{k}, \boldsymbol{u}\right)\right|}
$$

As $f_{\boldsymbol{U}}\left(\boldsymbol{u} \mid F_{i, k}\right)$ is known in closed form, it provides valuable information for setting an importance 303 sampling density function $f_{\boldsymbol{U}, I S}(\boldsymbol{u})$. In fact, the latter density function is selected as a weighted 304 summation of the probability density function associated with $\boldsymbol{u}$ conditioned on the different elementary 305 failure domains [55], that is:

$$
f_{\boldsymbol{U}, I S}(\boldsymbol{u})=\sum_{i=1}^{n_{\eta}} \sum_{k=1}^{n_{T}} w_{i, k} f_{\boldsymbol{u}}\left(\boldsymbol{u} \mid F_{i, k}\right)
$$

where $w_{i, k}$ is a weight associated with the elementary failure domain $F_{i, k}$, which is selected as [56]:

$$
w_{i, k}=\frac{P\left[F_{i, k}\right]}{\sum_{l=1}^{n_{\eta}} \sum_{m=1}^{n_{T}} P\left[F_{l, m}\right]}
$$

Taking into account all previous results, the importance sampling density function $f_{\boldsymbol{U}, I S}(\boldsymbol{u})$ reduces 308 to:

$$
f_{\boldsymbol{U}, I S}(\boldsymbol{u})=\frac{f_{\boldsymbol{U}}(\boldsymbol{u})}{\hat{P}_{f}} \sum_{i=1}^{n_{\eta}} \sum_{k=1}^{n_{T}}\left(1-F_{\chi_{n_{T}}^{2}}\left(C_{i, k}(\boldsymbol{u})^{2}\right)\right)
$$

The main advantage of the above importance sampling density function is that it ensures that samples 310 of the direction vector $\boldsymbol{u}$ which are highly relevant for the failure probability calculation are drawn 311 frequently within the context of simulation. 


\subsubsection{Estimator of first excursion probability}

The importance sampling density function proposed in Eq. (30) applied over Eq. (18) leads to the 314 following estimator for the first excursion probability:

$$
\tilde{P}_{f}^{\mathrm{DIS}} \approx \frac{\hat{P}_{F}}{N} \sum_{j=1}^{N} \frac{1-F_{\chi_{n_{T}}^{2}}\left(\left(C_{\min }\left(\boldsymbol{u}^{(j)}\right)\right)^{2}\right)}{D\left(\boldsymbol{u}^{(j)}\right)}
$$

where $\boldsymbol{u}^{(j)}$ are $\mathrm{N}$ independent, identically distributed samples that follow $f_{\boldsymbol{U}, I S}(\boldsymbol{u})$ and where:

$$
C_{\min }\left(\boldsymbol{u}^{(j)}\right)=\min \left(C_{i, k}\left(\boldsymbol{u}^{(j)}\right)\right)
$$

with $i=1, \ldots, n_{\eta}$ and $k=1, \ldots, n_{T}$, and:

$$
D\left(\boldsymbol{u}^{(j)}\right)=\sum_{i=1}^{n_{\eta}} \sum_{k=1}^{n_{T}}\left(1-F_{\chi_{n_{T}}^{2}}\left(C_{i, k}\left(\boldsymbol{u}^{(j)}\right)^{2}\right)\right)
$$

Samples of $\boldsymbol{u}$ distributed according to $f_{\boldsymbol{U}, I S}(\boldsymbol{u})$ can be generated using the approach described in 318 $[26,57]$. This approach comprises the following basic steps:

- Generate a sample $\boldsymbol{z}$ that follows a standard Gaussian distribution in $n_{T}$ dimensions.

- Select an elementary failure domain $F_{i^{\star}, k^{\star}}$ with probability proportional to its weight $w_{i^{\star}, k^{\star}}$.

- Generate a sample $\boldsymbol{z}^{\star}$ which follows a standard Gaussian distribution conditioned on the failure 322 event $F_{i^{\star}, k^{\star}}$. Such sample is generated by manipulating the projection of $\boldsymbol{z}$ onto $F_{i^{\star}, k^{\star}}$ [55].

- The sought sample of the direction is chosen as the unit vector pointing towards $\boldsymbol{z}^{\star}$, that is ${ }^{324}$ $\boldsymbol{u}=\boldsymbol{z}^{\star} /\left\|\boldsymbol{z}^{\star}\right\|_{2}^{2}$.

For a detailed description of the approach for generating a sample of $\boldsymbol{u}$ distributed according to $f_{\boldsymbol{U}, I S}(\boldsymbol{u}), \quad 326$ it is referred to $[26,57]$.

Numerical experience as reported in [26] suggests that the probability estimator in eq. (31) is highly ${ }_{328}$ efficient, as it allows estimating small failure probabilities $\left(10^{-3}\right.$ or less) with high accuracy (that is, 329 coefficient of variation smaller than 10\%) and high efficiency (that is, a few hundred samples). 


\section{Reliability of structures subjected to imprecise stochastic loading}

This section focuses on the calculation of the failure probability for the case where the effects of 332 imprecision are included in the description of the stochastic loading process by the interval vector $\boldsymbol{\theta}^{I}$. $\quad 333$ A naive approach would involve solving Eq. (13) and (14) directly following a double-loop approach. 334 However, since we consider linear structures, the double loop problem described by Eq. (13) and (14) 335 can be decoupled by determining those parameter values in $\boldsymbol{\theta}^{I}$ that yield $\bar{P}_{f}$ and $\underline{P}_{f}$ a priori using 336 operator norm theory.

\subsection{General framework}

The decoupling of the double loop is based on the operator norm theorem, which states that for 339 any continuous map $A: \mathbb{R}^{n_{K L}} \mapsto \mathbb{R}^{n_{T}}$ it holds that there exists a real number $c$ and arbitrary vector 340 $v \in \mathbb{R}^{n_{K L}}$ such that:

$$
\|A v\|_{p^{(1)}} \leq|c| \cdot\|v\|_{p^{(2)}}
$$

where $\|\bullet\|_{p}$ denotes a norm on the vector spaces $\mathbb{R}^{n_{K L}}$ and $\mathbb{R}^{n_{T}}$ and $p^{(i)} \geq 1$ constructs a particular $\mathcal{L}_{p} \quad{ }^{342}$ norm according to:

$$
\|v\|_{p}=\left(\sum_{i=1}^{n_{K L}}\left|v_{i}\right|^{p}\right)^{1 / p}
$$

Note that the norms on the vector spaces on both sides of the equation are not necessarily equal. 344 Physically speaking, Eq.(34) states that the length of the vector $v$ can maximally be increased by a 345

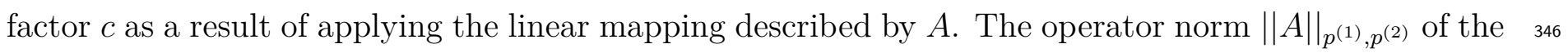
linear map $A$ describes how much $A$ lengthens vectors $v \in \mathbb{R}^{n_{K L}}$ in the maximum case, and is defined 347 as:

$$
\|A\|_{p^{(1)}, p^{(2)}}=\inf \left\{c \geq 0:\|A v\|_{p^{(1)}} \leq|c| \cdot\|v\|_{p^{(2)}} \quad \forall v \in \mathbb{R}^{n_{K L}}\right\}
$$

The operator norm on $A$ can equivalently be defined as:

$$
\|A\|_{p^{(1)}, p^{(2)}}=\sup \left\{\frac{\|A v\|_{p^{(1)}}}{\|v\|_{p^{(2)}}}: v \in \mathbb{R}^{n_{K L}} \text { with } v \neq 0\right\}
$$

Note that the definition of the vector spaces as $\mathbb{R}^{n_{K L}}$ and $\mathbb{R}^{n_{T}}$ is only made to highlight the link 350 with the previous section. In fact, this theorem holds for all normed spaces on $\mathbb{R}$ or $\mathbb{C}$, as long as the 351 map $A$ is continuous.

In the context of determining the bounds on the probability of failure of a linear structure, subjected 353 
to an imprecise stochastic load, $A_{i}$ is defined as $A_{i}(\boldsymbol{\theta})=\left[\boldsymbol{a}_{i, 1}^{T}(\boldsymbol{\theta}) ; \boldsymbol{a}_{i, 2}^{T}(\boldsymbol{\theta}) ; \ldots ; \boldsymbol{a}_{i, n_{T}}^{T}(\boldsymbol{\theta})\right]$, i.e., the column 354 stacking of the $\boldsymbol{a}_{i, k}$ vectors for the $k=1, \ldots, n_{T}$ time instants (as defined in Eq. (10)). In this case, 355 Eq. (34) can be rewritten as:

$$
\left\|A_{i}(\boldsymbol{\theta}) \boldsymbol{z}\right\|_{p^{(1)}} \leq\left|c_{i}(\boldsymbol{\theta})\right| \cdot\|\boldsymbol{z}\|_{p^{(2)}}
$$

which is by virtue of Eq. (8) equivalent to:

$$
\left\|\eta_{i}(t, \boldsymbol{\theta}, \boldsymbol{z})\right\|_{p^{(1)}} \leq\left|c_{i}(\boldsymbol{\theta})\right| \cdot\|\boldsymbol{z}\|_{p^{(2)}}
$$

where $\eta_{i}(t, \boldsymbol{z})$ denotes the $i^{t h}$ dynamic response as a function of $t$, and $\boldsymbol{z}$ are the i.i.d. Gaussian variables 358 stemming from the KL expansion in Eq. (5). The computation of the operator norm $\|A\|_{p^{(1)}, p^{(2)}}$ is in this 359 case related to the choice of the type of $\mathcal{L}_{p}$ norm that is selected on both sides of the equation, which is 360 highly case-dependent. In the specific case of bounding the probability of failure described as the first 361 excursion probability of a linear dynamic system, the analysis is mainly driven by the extreme responses 362 $\eta_{i}(t, \boldsymbol{\theta}, \boldsymbol{z})$ within the duration $T$ of the stochastic excitation. As such, the operator norm should be 363 defined such that it describes the maximum amplification of the length of $\boldsymbol{z}$ towards the maximum 364 values in $\eta_{i}(t, \boldsymbol{\theta}, \boldsymbol{z})$, as those drive the calculation of the first excursion probability. Therefore, following 365 problem is considered:

$$
\left\|\eta_{i}(t, \boldsymbol{\theta}, \boldsymbol{z})\right\|_{\infty} \leq\left|c_{i}(\boldsymbol{\theta})\right| \cdot\|\boldsymbol{z}\|_{2}
$$

The choice for an $\mathcal{L}_{\infty}$ norm is motivated by the notion that those values in $\boldsymbol{\theta}$ that yield the most 367 extreme structural responses are of highest interest, as these extremes in the responses are the ones 368 that predominantly drive the probability of failure. Concerning the right hand side, the $\mathcal{L}_{2}$ norm is 369 selected as it can be loosely defined as the energy content in the random variables. It should be noted 370 that the latter is in fact a constant value (in average), as $\boldsymbol{z}$ is by construction a set of i.i.d. standard 371 normal random variables. As a matter of fact, the selection of the norm on $\|\boldsymbol{z}\|$ does not matter, as the 372 vector in case of a Gaussian stochastic process is always dictated by a standard normal distribution (as 373 presented in the context of this work). In this case, it can be shown that $\|A\|_{p^{(1)}, p^{(2)}}$ can be computed 374 as [58]:

$$
\|A\|_{p^{(1)}, p^{(2)}}=\max _{l}\left\|A_{i, l:}(\boldsymbol{\theta})\right\|_{2}
$$

where the subscript $l$ : denotes taking the $l^{\text {th }}$ row of the matrix $A_{i}(\boldsymbol{\theta})$. As such, $\|A\|_{p^{(1)}, p^{(2)}}$ is computed 376 as the maximum $\mathcal{L}_{2}$ norm of a row of $A_{i}(\boldsymbol{\theta})$. Physically speaking, $\boldsymbol{\theta}$ is a measure for the best possible 377 
amplification of the energy contained in $\boldsymbol{z}$ to the extreme values of the dynamic response $\eta_{i}(t, \boldsymbol{\theta}, \boldsymbol{z}) \quad 378$ under consideration.

\subsection{Bounds on the probability of failure using operator norm theory}

To determine the bounds on $P_{f}$ without resorting to a double loop optimization procedure, the 381 operator norm framework that was explained in the previous section can be applied. Indeed, since the 382 operator norm describes the elongation of $\boldsymbol{z}$ to the largest responses of the system, it can be used to 383 determine those values of $\boldsymbol{\theta}$ that provide the largest possible elongation of $\boldsymbol{z}$ by $\min / \mathrm{maximizing}$ the 384 operator norm with respect to $\boldsymbol{\theta}$.

First, consider a structure with a single dynamic response $\eta(t, \boldsymbol{z})$ that drives the computation of 386 $P_{f}$. In this case, to determine which values in $\boldsymbol{\theta}^{I}$ yield respectively $\bar{P}_{f}$ and $\underline{P}_{f}$, following optimization 387 problems have to be solved:

$$
\begin{aligned}
& \theta^{*}=\underset{\boldsymbol{\theta} \in \boldsymbol{\theta}^{I}}{\operatorname{argmin}} \max _{l}\left\|A_{l:}(\boldsymbol{\theta})\right\|_{2} \\
& \theta^{\bar{*}}=\underset{\boldsymbol{\theta} \in \boldsymbol{\theta}^{I}}{\operatorname{argmax}} \max _{l}\left\|A_{l:}(\boldsymbol{\theta})\right\|_{2}
\end{aligned}
$$

In case more than one dynamic response $\eta_{i}(t, \boldsymbol{z}), i=1, \ldots, n_{\eta}$ is considered, the optimization problem 389 has to be expanded. Indeed, in this case a matrix $A_{i}$ is defined for each response of interest, yielding 390 following optimization problems:

$$
\begin{aligned}
& \theta^{*}=\underset{\boldsymbol{\theta} \in \boldsymbol{\theta}^{I}}{\operatorname{argmin}} \max _{i=1, \ldots, n_{\eta}} \max _{l}\left\|A_{i, l:}(\boldsymbol{\theta})\right\|_{2} \\
& \theta^{\bar{*}}=\underset{\boldsymbol{\theta} \in \boldsymbol{\theta}^{I}}{\operatorname{argmax}} \max _{i=1, \ldots, n_{\eta}} \max _{l}\left\|A_{i, l:}(\boldsymbol{\theta})\right\|_{2}
\end{aligned}
$$

In this equation, the maximal values of $\max _{l}\left\|A_{i, l:}(\boldsymbol{\theta})\right\|_{2}$ over all responses $\eta_{i}(t, \boldsymbol{z}), i=1, \ldots, n_{\eta}$ have 392 to be considered jointly. Since usually only a limited number of dynamic responses are considered, the 393 additional computational cost of the inner optimization is trivial.

These analyses show that the parameters of the stochastic ground acceleration model that yield the 395 bounds on the first excursion probability of the structure can be determined in two optimization calls. 396 Furthermore, this only requires a single deterministic call to the FE solver, namely to determine the 397 impulse response functions $h_{i}$ that are required to assemble $\boldsymbol{a}_{i, k}$, as shown in Eq. (10). Therefore, since 398 the interval problem corresponding to Eq. (13) and Eq. (14) can be solved completely a priori, only 2 399 
computations of the probability of failure are required:

$$
\underline{P}_{f}=\int_{\boldsymbol{z} \in \mathbb{R}^{n} K L} I_{F}\left(\boldsymbol{z}, \boldsymbol{\theta}^{*}\right) f_{\boldsymbol{Z}}(\boldsymbol{z}) d \boldsymbol{z}
$$

to obtain the lower bound and:

$$
\bar{P}_{f}=\int_{\boldsymbol{z} \in \mathbb{R}^{n} K L} I_{F}\left(\boldsymbol{z}, \boldsymbol{\theta}^{\bar{*}}\right) f_{\boldsymbol{Z}}(\boldsymbol{z}) d \boldsymbol{z}
$$

to obtain the upper bound, strongly reducing the computational cost of the determination of the bounds 402 on the first excursion probability of the structure subjected to an imprecise stochastic ground motion 403 acceleration. As such, instead of having to solve $2 \times n_{\text {opt }} \times n_{P_{f}}$ solutions of Eq. (8), only $2 \times\left(n_{\text {opt }}+n_{P_{f}}\right) \quad{ }_{404}$ solutions are required, where $n_{\text {opt }}$ denotes the number of function calls performed by the optimization 405 algorithm to solve the 'outer' loop, while $n_{P_{f}}$ denotes the number of simulations required for determining 406 the failure probability in the 'inner' loop.

\section{Case study 1: Single-degree-of-freedom oscillator}

\subsection{Case introduction}

This example comprises a single-degree-of-freedom oscillator with mass $m=1 \mathrm{~kg}$, stiffness $k=225 \quad 410$ $\mathrm{N} / \mathrm{m}$ and classical damping $d=5 \%$ subject to a stochastic ground acceleration $g_{A}(t)$. The ground 411 acceleration follows a modulated Clough-Penzien (CP) model. A description of this model along with the ${ }_{412}$ modulating function considered can be found in Appendix A. Nominal parameters for the modulated ${ }_{413}$ Clough-Penzien model are set equal to $\left[\omega_{g}, \omega_{f}, \zeta_{g}, \zeta_{f}, S_{0}, c_{1}, c_{2}\right]=\left[6 \pi, 0.6 \pi, 0.6,0.6,4 \times 10^{-2}, 0.14,0.16\right]$. ${ }_{414}$ The total duration of the acceleration is $20[\mathrm{~s}]$ and the time step discretization is $\Delta t=0.01 \mathrm{~s}$. The ${ }_{415}$ prescribed threshold level is $b=0.1 \mathrm{~m}$. The oscillator is at rest at the beginning of the stochastic ${ }_{416}$ excitation. The K-L expansion of the stochastic process is truncated at $99 \%$ of the total variance, ${ }_{417}$ yielding approximately 1300 terms in the expansion. The exact number of terms in the expansion ${ }_{418}$ depends on the actual parameter values in $\boldsymbol{\theta}^{I}$, and calculated for each stochastic process propagation ${ }_{419}$ run separately based on the variance truncation. Directional importance sampling with a sample size ${ }_{420}$ of 500 deterministic model evaluations is used to compute the crisp probability of failure. Using this ${ }_{421}$ set of parameters, the probability of failure of the mass-spring system is 0.0053 with a coefficient of ${ }^{422}$ variation of 0.0359 .

To illustrate the performance of the developed approach, a study is performed with wide interval ${ }_{424}$ 
widths on the parameters in $\boldsymbol{\theta}^{I}$, as illustrated in table 1 . These bounds are derived from the data in 425 table A.7 and expert knowledge and correspond to a case of nearly non-informative estimates on the ${ }_{426}$ parameters. For the soil conditions, parameters spanning the full range between Soft and Firm soil are ${ }_{427}$ considered. Due to the interval-valued definition of these parameters, also the probability of failure of 428 the model will become interval valued. Furthermore, since the intervals on the parameters are wide, it 429 is expected that the upper and lower bound on $P_{f}$ will diverge also significantly.

Table 1: Tested values for $\boldsymbol{\theta}^{I}$

\begin{tabular}{ccccccc}
\hline$\omega_{g}^{I}$ & $\omega_{f}^{I}$ & $\zeta_{g}^{I}$ & $\zeta_{f}^{I}$ & $S_{0}^{I}$ & $c_{1}^{I}$ & $c_{2}^{I}$ \\
\hline$\pi[2.4 ; 8]$ & $\pi[0.24 ; 0.8]$ & {$[0.6 ; 0.85]$} & {$[0.6 ; 0.85]$} & $4 \times 10^{-2}[0.75 ; 1.25]$ & {$[0.12 ; 0.16]$} & {$[0.14 ; 0.18]$} \\
\hline
\end{tabular}

\subsection{Computation of the bounds on $P_{f}$}

The methods developed in this paper are applied to compute the bounds on $P_{f}$. Hereto, the operator ${ }_{432}$ norm that represents the magnitude of the amplification of the stochastic base excitation of the SDOF oscillator towards the maximally occurring displacement values is computed as:

$$
\|A(\boldsymbol{\theta})\|_{p^{(1)} \rightarrow \infty, p^{(2)}=2}=\max _{l}\left\|\boldsymbol{A}_{l:}(\boldsymbol{\theta})\right\|_{2}
$$

with $\boldsymbol{A}=\left[\boldsymbol{a}_{1}^{T}, \boldsymbol{a}_{2}^{T}, \ldots, \boldsymbol{a}_{n_{T}}^{T}\right]$ a matrix collecting the $n_{T}$ vectors $\boldsymbol{a}_{k}$ (see eq. (10)) for each time instant 435 $t_{k}$ of the simulation. The parameter values of $\boldsymbol{\theta}^{I}$ that yield the bounds on $P_{f}$ are then determined 436 by solving Eq. (42) and Eq.(43). These optimization problems are solved using a sequential quadratic ${ }_{437}$ programming approach. Solving this equation leads to the intervals for the parameters $\boldsymbol{\theta}^{I, *}=\left[\boldsymbol{\theta}^{*} ; \boldsymbol{\theta}^{*}\right] \quad{ }^{438}$ shown in table 3. The probability of failure corresponding to these two sets of hyper-parameters is 439 then computed via Eq. (46) and Eq. (47). As such, only two calculations of the probability of failure 440 are required, and hence, 1000 deterministic model evaluations. As can be noted from table 3, some 441 values degenerate to a crisp number, indicating that both extremes of the maximum amplification of 442 the signal, and hence, the probability of failure depend on the same vertex of $\boldsymbol{\theta}^{I}$. Furthermore, it may ${ }_{443}$ be noted that the upper bound on $P_{f}$ depends on a value of $\omega_{g}^{I}$ that is located at neither bound.

To validate this approach, two additional methods for computing the bounds of $P_{f}$ of the SDOF ${ }_{445}$ oscillator subjected to the imprecise stochastic load are applied: a vertex analysis and a double-loop 446 Quasi Monte Carlo simulation. Both methods are aimed at replacing the 'outer' optimization loop in 447 Eq. (13) and Eq. (14) by a computationally more efficient approximation. 
The vertex analysis replaces the outer optimization in Eq. (13) and Eq. (14) by assessing all possible 449 combinations of the bounds of the parameters in $\boldsymbol{\theta}^{I}$. For each of these combinations, a value for $P_{f}$ is 450 computed. Hence, $2^{7}=128$ computations of the probability of failure are required and hence, 64000451 deterministic model evaluations.

The Quasi Monte Carlo simulation approach replaces the outer optimization problems by means of ${ }_{453}$ a quasi-random sampling scheme under the assumption of a uniform distribution between the bounds ${ }_{454}$ in $\boldsymbol{\theta}^{I}$. Following these auxilliary uniform distributions, a Sobol sequence with 500 points is generated ${ }^{455}$ and $P_{f}$ is computed for each of these samples. This leads to 500 computations of the probability of ${ }_{456}$ failure and hence, 250000 deterministic model evaluations.

The results of these three propagation schemes are shown in Table 2. As can be noted, the bounds ${ }_{458}$ obtained by the optimization procedure are wider as compared to both the sets of bounds obtained via 459 vertex analysis and Sobol sampling. Both the vertex analysis and Sobol sampling underestimate the 460 upper bound on the probability of failure significantly. Furthermore, the lower bound of $P_{f}$ as computed 461 by both the optimization procedure and the vertex analysis is lower as compared to the one obtained ${ }_{462}$ by the Sobol set analysis. However, $\underline{P}_{f}$ as computed by the vertex analysis is lower as compared to 463 the result of the Optimization procedure. This is explained by the results in table 3, which shows that 464 the optimization method apparently derives the same value for the lower bound as the corresponding 465 vertex. However, due to numerical precision of the optimizer, both values differ very slightly, and 466 hence, small differences in the resulting failure probability exist, especially since the lower bound is ${ }^{467}$ extremely low. Increasing the numerical accuracy of the optimization algorithm that is used to solve 468 Eq. (43) will alleviate this problem. Also from this table, it is clear that the problem is not necessarily ${ }_{469}$ monotonic with respect to the parameters of the CP model. Indeed, looking at the values that yield the 470 upper bound, one can see that the value for $\omega_{g}$ that gives the highest probability of failure lies inside ${ }^{471}$ the interval. Since the vertex approach assumes monotonicity of all parameters with respect to $P_{f}$, it 472 is not capable of determining the correct upper bound for the failure probability. The optimization 473 approach introduced in this paper however, does not make assumptions on the monotonicity of $P_{f}$ with 474 respect to any parameters, and is therefore capable of determining the bounds exactly, at far reduced 475 computational cost as compared to the other two methods. The origin of this non-monotonicity lies 476 in the interplay between the frequency content of the non-stationary stochastic base excitation with 477 resonances inside the structure. Since the optimization method takes this into account (see section 4), 478 it is capable of tackling the non-monotonicity. This point is further clarified in figure 3, which shows the 479 
Clough-Penzien power spectra corresponding to $\boldsymbol{\theta}^{\bar{*}}$, obtained by the Operator norm optimization method 480 (O), Vertex sampling (V) and Sobol set propagation (S) together with the damped natural frequency 481 of the SDOF oscillator $\left(\omega_{d}\right)$. As is clear, the dominant frequency of the CP spectrum corresponding 482 to $\boldsymbol{\theta}^{\bar{*}}$ obtained via optimizing the operator norm perfectly matches with the damped eigenfrequency 483 of the oscillator, which causes resonance, and hence, a higher probability of failure. This match with 484 the eigenfrequency of the oscillator is not present in the two other spectra. It should be noted that 485 this method only works when the considered structure is linear due to the inherent coupling with the 486 solution of a dynamic linear system that underlies the derivations in section 4.

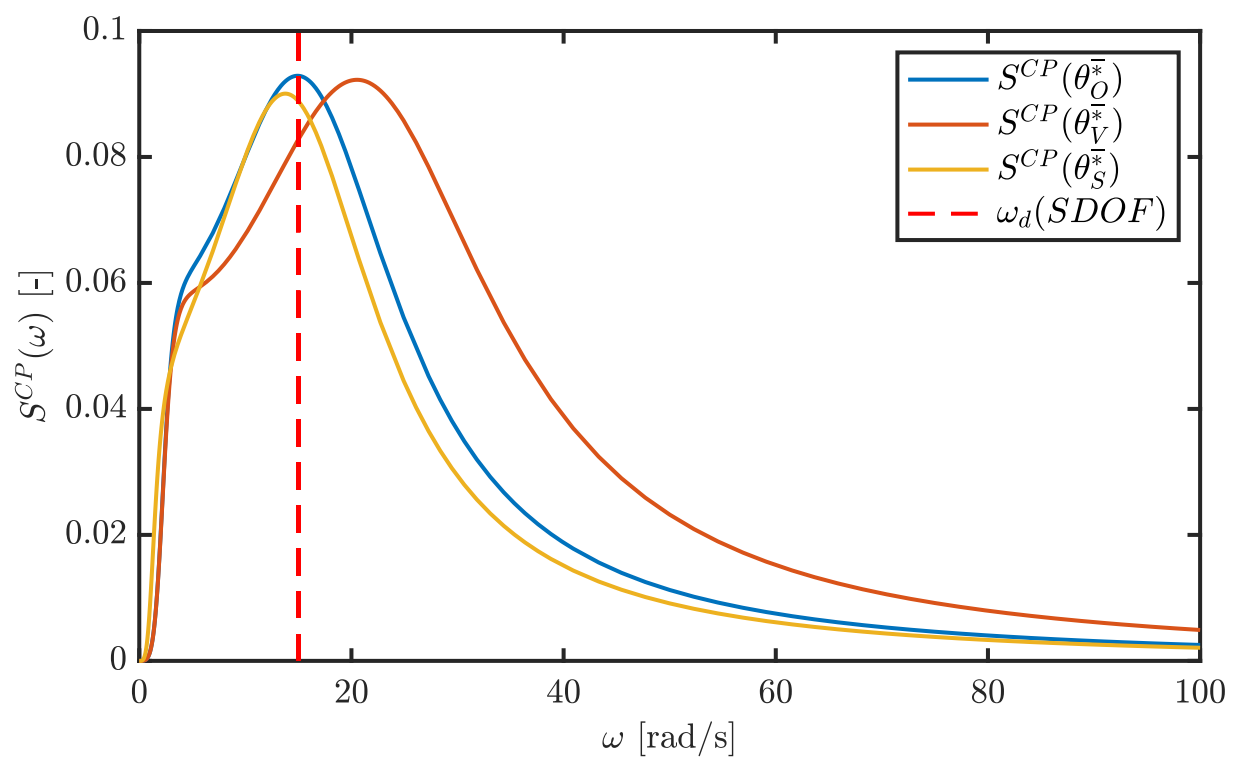

Figure 3: Clough-Penzien power spectra corresponding to $\boldsymbol{\theta}^{\bar{*}}$, obtained by the Operator norm optimization method (O), Vertex sampling (V) and Sobol set propagation $(\mathrm{S})$.

Table 2: Computed bounds on the failure probability

\begin{tabular}{cccc}
\hline & Vertex analysis & Sobol sampling & Optimization \\
\hline$P_{f}$ & {$\left[2.77 \times 10^{-15} ; 0.018\right]$} & {$\left[4.7 \times 10^{-14} ; 0.0192\right]$} & {$\left[2.78 \times 10^{-15} ; 0.328\right]$} \\
$\max _{l}\left\|A_{l:}(\boldsymbol{\theta})\right\|_{2}$ & {$[0.01186 ; 0.0272]$} & {$[0.0123 ; 0.0279]$} & {$[0.0118 ; 0.0286]$} \\
\hline
\end{tabular}

Table 3: Identified values for $\boldsymbol{\theta}^{I, *}$. O are the results from the optimization procedure, whereas $\mathrm{V}$ denotes the results from the vertex method. Note that the bounds on the reported intervals are those values that correspond to a bound on $P_{f}$.

\begin{tabular}{cccccccc}
\hline Method & $\omega_{g}^{I}$ & $\omega_{f}^{I}$ & $\zeta_{g}^{I}$ & $\zeta_{f}^{I}$ & $S_{0}^{I}$ & $c_{1}^{I}$ & $c_{2}^{I}$ \\
\hline $\mathrm{O}$ & $\pi[2.4 ; 5.84]$ & $\pi[0.8 ; 0.8]$ & {$[0.85 ; 0.6]$} & {$[0.85 ; 0.6]$} & $4 \times 10^{-2}[0.75 ; 1.25]$ & {$[0.16 ; 0.12]$} & {$[0.18 ; 0.14]$} \\
$\mathrm{V}$ & $\pi[2.4 ; 8]$ & $\pi[0.8 ; 0.8]$ & {$[0.85 ; 0.6]$} & {$[0.85 ; 0.6]$} & $4 \times 10^{-2}[0.75 ; 1.25]$ & {$[0.16 ; 0.12]$} & {$[0.18 ; 0.14]$} \\
\hline
\end{tabular}

A further illustration of the results is given in figure 4. This figure shows the value of $P_{f}$ plotted ${ }^{488}$ against $\max _{l}\left\|A_{l:}(\boldsymbol{\theta})\right\|_{2}$ for 500 Sobol samples in between the bounds of $\boldsymbol{\theta}^{I}$, combined with the data from 489 
the vertex analysis. It can be noted that a monotonic relationship between these two quantities exists, 490 and furthermore that the bounds on $P_{f}$ indeed correspond to the bounds on $\max _{l}\left\|A_{l:}(\boldsymbol{\theta})\right\|_{2}$. Finally, it 491 can be noted that also a few Sobol samples lie outside the bounds provided by the vertex method (e.g., 492 one around $\max _{l}\left\|A_{l:}(\boldsymbol{\theta})\right\|_{2}=0.028$.

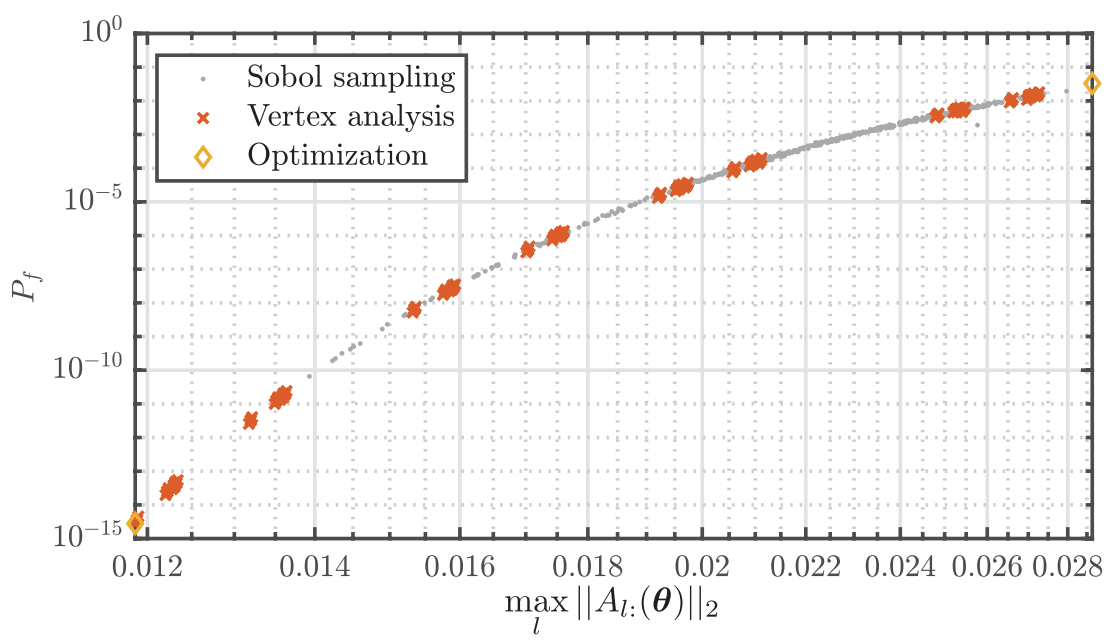

Figure 4: $P_{f}$ plotted against $\max _{l}\left\|A_{l:}(\boldsymbol{\theta})\right\|_{2}$ for 500 Sobol samples in between the bounds of $\boldsymbol{\theta}^{I}$, combined with the data from the vertex analysis

\section{Case study 2: six story building model}

\subsection{Case introduction}

The second example involves the six story reinforced concrete building model depicted in Figure 5, which is borrowed from [59]. Each floor plan is of square shape with side length $32 \mathrm{~m}$ and story height of $3.6 \mathrm{~m}$. All floor slabs possess a thickness of $20 \mathrm{~cm}$ and are supported by a C-shaped shear ${ }_{498}$ wall of $20 \mathrm{~cm}$ thickness and 16 columns of square cross section with side length $40 \mathrm{~cm}$. The Young's 499 modulus is set equal to $2.3 \times 10^{10} \mathrm{~Pa}$. It is assumed that the building undergoes small displacements 500 and hence, it is modeled as linear elastic. The behavior of the building is characterized by means of 501 a finite element model that comprises about 9500 shell and beam elements and more than $50 \times 10^{3} 502$ degrees-of-freedom. The building is excited by a stochastic ground acceleration along the $y$ direction. ${ }^{503}$ This ground acceleration is generated considering a modulated Clough-Penzien model, with nominal 504 parameters $\left[\omega_{g}, \omega_{f}, \zeta_{g}, \zeta_{f}, S_{0}, c_{1}, c_{2}\right]=\left[4 \pi, 0.4 \pi, 0.7,0.7,3 \times 10^{-4}, 0.14,0.16\right]$. Similarly to example 1 , the 505 stochastic process is truncated at $99 \%$ of the total variance, yielding approximately 1300 terms in 506 the KL expansion. The total duration of the acceleration is $20 \mathrm{~s}$ and the time step discretization is 507 $\Delta t=0.01 \mathrm{~s}$. Due to design purposes, it is of interest to control that the interstory drifts along the $y$ 508 
direction does not exceed a threshold level of $2 \times 10^{-3}$ times the story height. These interstory drifts 509 are controlled at five points, between nodes $n_{2}-n_{1}, n_{3}-n_{2}, n_{4}-n_{3}, n_{5}-n_{4}$ and $n_{6}-n_{5}$. The probability of 510 failure is computed with Direction Importance Sampling with a sample size of 500 deterministic model 511 evaluations. Table 4 illustrates the parameter interval on the parameters of the CP ground acceleration 512 that is used to model the imprecision in the stochastic ground acceleration. Also in this case, due to 513 the imprecision in the stochastic load, represented by the interval-valued definition of the governing 514 hyper-parameters $\boldsymbol{\theta}$, the probability of failure will become interval-valued as well.

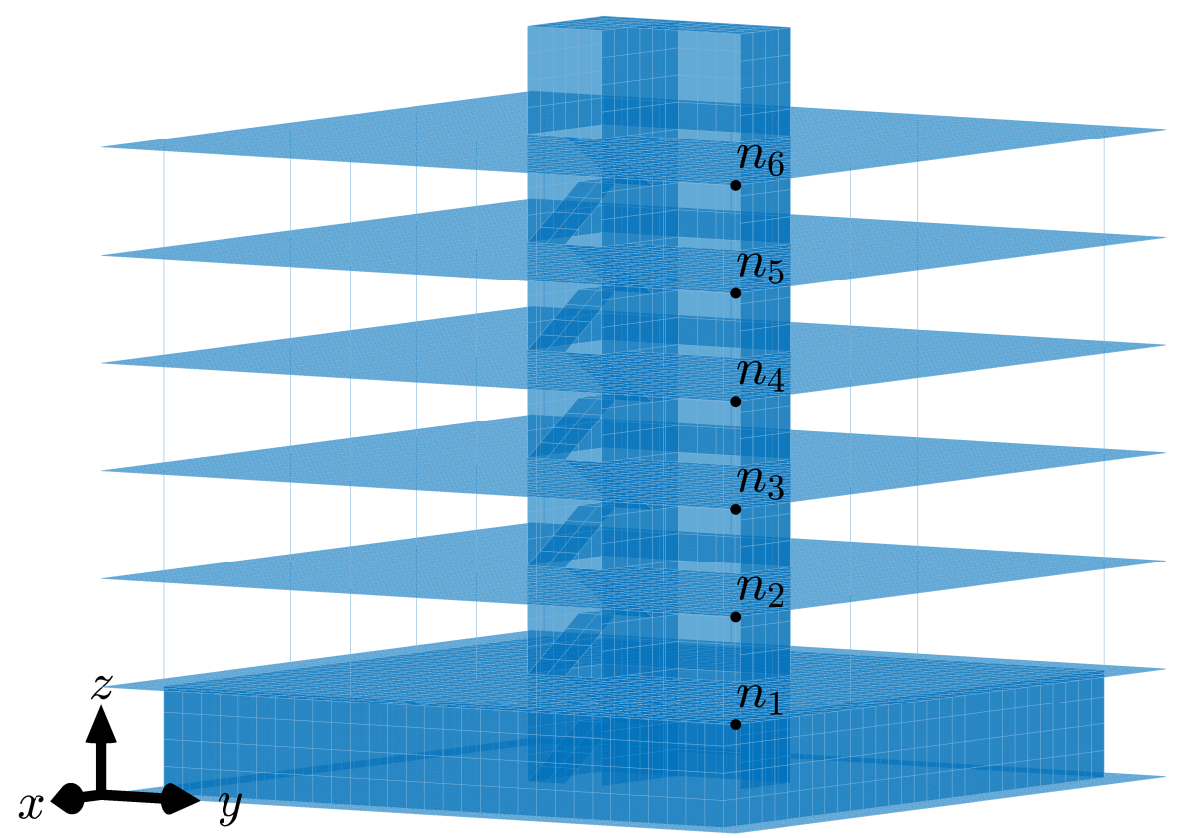

Figure 5: Example 2 - Isometric view of the building model

Table 4: Tested values for $\boldsymbol{\theta}^{I}$

\begin{tabular}{ccccccc}
\hline$\omega_{g}^{I}$ & $\omega_{f}^{I}$ & $\zeta_{g}^{I}$ & $\zeta_{f}^{I}$ & $S_{0}^{I}$ & $c_{1}^{I}$ & $c_{2}^{I}$ \\
\hline$\pi[2.4 ; 8]$ & $\pi[0.24 ; 0.8]$ & {$[0.6 ; 0.85]$} & {$[0.6 ; 0.85]$} & $3 \times 10^{-4}[0.75 ; 1.25]$ & {$[0.12 ; 0.16]$} & {$[0.14 ; 0.18]$} \\
\hline
\end{tabular}

\subsection{Computation of the bounds on $P_{f}$}

The results of these three propagation schemes are reported in table 5 . First, it can be noted that the bounds produced by the Sobol sampling underestimate the width of the interval on $P_{f}$ severely, where especially the underestimation of $\bar{P}_{f}$ is problematic. Furthermore, comparing the results of the vertex 
analysis with the optimization procedure, it can be concluded that both predict the same bounds on 520 the probability of failure. As such, in this case, the problem behaves seemingly monotonic with respect ${ }_{521}$ to $\boldsymbol{\theta}$.

Furthermore, despite the fact that the intervals on $\boldsymbol{\theta}^{I}$ are the same as in the case with the SDOF ${ }_{523}$ oscillator, the realizations that yield respectively $\underline{P}_{f}$ and $\bar{P}_{f}$ are completely different. For instance, 524 concerning $\omega_{g}^{I}$, the values that give $\underline{P}_{f}$ in the SDOF oscillator give $\bar{P}_{f}$ on the building model. This 525 is due to the fact that the bounds of $P_{f}$ are determined by both the energy content of the stochastic ${ }_{526}$ excitation, as well as the match of its dominant frequencies of the excitation with the resonances 527 of the structure under consideration, especially when the latter are dominated by a few eigenmodes. 528 Also this example shows that the proposed approach is capable of providing an analyst with a highly 529 accurate estimation of the bounds on $P_{f}$ within a very limited computational budget, as compared to 530 a double loop approach. Especially the gain in computational efficiency is noteworthy, although the 531 vertex analysis and optimization approach yield the same results concerning the bounds, the latter 532 approach needs only $1+2 \times 500=1001$ deterministic model evaluations, whereas the former requires 533 $128 \times 500=64000$ deterministic model evaluations, without any guarantee of conservatism in case the 534 relation between $\boldsymbol{\theta}^{I}$ and $P_{f}$ is not strictly monotonic.

Table 5: Computed bounds on the failure probability

\begin{tabular}{cccc}
\hline & Vertex analysis & Sobol sampling & Optimization \\
\hline$P_{f}$ & {$\left[4.47 \times 10^{-07} ; 0.0495\right]$} & {$\left[1.14 \times 10^{-06} ; 0.0275\right]$} & {$\left[4.47 \times 10^{-07} ; 0.0495\right]$} \\
$\max _{i} \max _{l}\left\|A_{i, l:}(\boldsymbol{\theta})\right\|_{2}$ & {$\left[9.44 \times 10^{-04} ; 2.34 \times 10^{-03}\right]$} & {$\left[9.78 \times 10^{-04} ; 2.21 \times 10^{-03}\right]$} & {$\left[9.44 \times 10^{-04} ; 2.34 \times 10^{-03}\right]$} \\
\hline
\end{tabular}

Table 6: Identified values for $\boldsymbol{\theta}^{I, *}$

\begin{tabular}{cccccccc}
\hline Method & $\omega_{g}^{I}$ & $\omega_{f}^{I}$ & $\zeta_{g}^{I}$ & $\zeta_{f}^{I}$ & $S_{0}^{I}$ & $c_{1}^{I}$ & $c_{2}^{I}$ \\
\hline $\mathrm{O}$ & $\pi[8 ; 2.4]$ & $\pi[0.8 ; 0.8]$ & {$[0.85 ; 0.6]$} & {$[0.85 ; 0.6]$} & $3 \times 10^{-4}[0.75 ; 1.25]$ & {$[0.16 ; 0.12]$} & {$[0.18 ; 0.14]$} \\
$\mathrm{V}$ & $\pi[8 ; 2.4]$ & $\pi[0.8 ; 0.8]$ & {$[0.85 ; 0.6]$} & {$[0.85 ; 0.6]$} & $3 \times 10^{-4}[0.75 ; 1.25]$ & {$[0.16 ; 0.12]$} & {$[0.18 ; 0.14]$} \\
\hline
\end{tabular}

Finally, figure 6 shows the behavior of $\max _{i=1, \ldots, n_{\eta}} \max _{l}\left\|A_{i, l:}(\boldsymbol{\theta})\right\|_{2}$ with respect to $P_{f}$. Specifically, 536 this figure shows the combined results of the optimization procedure, Sobol sampling and vertex analysis. 537 It can be noted that also in this case a sufficiently convex optimization problem is obtained. However, 538 the functional relation is comparably less smooth that in the case where only one response is considered. 539 This is due to the additional optimization layer in Eq. (45). 


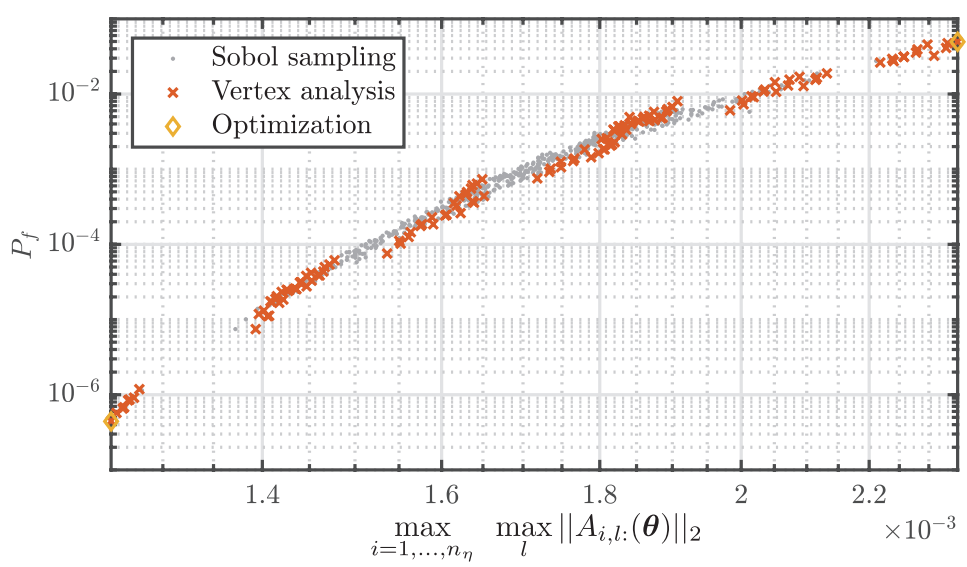

Figure 6: $P_{f}$ plotted against $\max _{i=1, \ldots, n_{\eta}} \max _{l}\left\|A_{i, l:}(\boldsymbol{\theta})\right\|_{2}$ for 500 Sobol samples in between the bounds of $\boldsymbol{\theta}^{I}$, combined with the data from the vertex analysis and the optimization procedure

\section{Conclusions}

This paper presents a highly efficient and accurate approach to determine the bounds on the first ex- 542 cursion probability of a linear oscillating system when the structure is excited by an imprecise stochastic 543 process. The method decouples the epistemic uncertainty from the aleatory by a priori determining 544 which parameters in the stochastic spectrum yield the bounds on the probability of failure of the struc- 545 ture. As such, the propagation of the epistemic uncertainty only requires a single deterministic model 546 evaluation together with the solution of two optimization problems. The aleatory uncertainty in the 547 problem is propagated using the framework of Directional Importance Sampling. Using the two param- 548 eter sets yielding the bounds on the probability of failure, only two Directional Importance Sampling 549 evaluations are required.

The method is illustrated using the propagation of a non-stationary modulated Clough-Penzien 551 excitation spectrum, but is generally applicable to the case of linear dynamical systems subjected to 552 imprecise stochastic excitation. The conclusions of the paper can be summarized as follows:

- the proposed technique provides an extremely efficient approach to determine the bounds on the 554 first excursion probability of a linear oscillator subjected to an imprecise stochastic excitation 555

- the method is shown to be more accurate than existing double loop approaches such as double 556 loop quasi Monte Carlo

- since the method does not make assumptions on the monotonicity of the relationship of the 


\section{Acknowledgment}

The Research Foundation Flanders is gratefully acknowledged for the support of Matthias Faes under 562 grant number 12P3519N. Marcos Valdebenito acknowledges the support of ANID (National Agency for 563 Research and Development, Chile) under its program FONDECYT, grant number 1180271; Universidad 564 Tecnica Federico Santa Maria under its program PAC (Programa Asistente Cientifico 2017); and the 565 Alexander von Humboldt Foundation through its program Humboldt Research Fellowship for Experienced 566 Researchers.

\section{Appendix A. Clough-Penzien model}

One of the most commonly used parametric models for the power spectral density associated with 569 ground acceleration is the Kanai-Tajimi spectrum (see, e.g. [32]), whose physical basis consists of a 570 white noise process of spectral intensity $S_{0}$ associated with the bedrock excitation that passes through 571 a linear soil filter characterized in terms of a natural frequency $\omega_{g}$ and damping $\zeta_{g}$. A drawback of the 572 Kanai-Tajimi spectrum is that its associated velocity and displacement power spectra are not defined 573 as the circular frequency tends to zero $(\omega \rightarrow 0)$. Such issue is remedied by the Clough-Penzien power 574 spectrum, which passes the signal produced by the Kanai-Tajimi spectrum through an additional linear 575 filter with natural frequency $\omega_{f}$ and damping $\zeta_{f}$. The expression for the Clough-Penzien power spectrum 576 $S^{\mathrm{CP}}$ is given by $[32,60]$ :

$$
S^{\mathrm{CP}}(\omega)=\frac{\omega_{g}^{4}+\left(2 \zeta_{g} \omega_{g} \omega\right)^{2}}{\left(\omega_{g}^{2}-\omega^{2}\right)^{2}+\left(2 \zeta_{g} \omega_{g} \omega\right)^{2}} \cdot \frac{\omega^{4}}{\left(\omega_{f}^{2}-\omega^{2}\right)^{2}+\left(2 \zeta_{f} \omega_{f} \omega\right)^{2}} \cdot S_{0}
$$

Typical values for the filter parameters associated with the Clough-Penzien power spectrum as suggested in [1] are shown in Table A.7. The autocorrelation function $R^{C P}(\tau)$ associated with the Clough-Penzien

\begin{tabular}{|c|c|c|c|c|}
\hline Soil type & $\omega_{g}[\mathrm{rad} / \mathrm{s}]$ & $\zeta_{g}$ & $\omega_{f}[\mathrm{rad} / \mathrm{s}]$ & $\zeta_{f}$ \\
\hline Firm & $8 \pi$ & 0.60 & $0.8 \pi$ & 0.60 \\
\hline Medium & $5 \pi$ & 0.60 & $0.5 \pi$ & 0.60 \\
\hline Soft & $2.4 \pi$ & 0.85 & $0.24 \pi$ & 0.85 \\
\hline
\end{tabular}

Table A.7: Filter parameters associated with Clough-Penzien power spectrum 
power spectrum is calculated taking the inverse Fourier transform of $S^{\mathrm{CP}}$, yielding [61]:

$$
\begin{aligned}
R^{\mathrm{CP}}(\tau) & =\frac{\pi S_{0}}{2 \zeta_{g} \omega_{g}} e^{-\zeta_{g} \omega_{g}|\tau|}\left(\left(C_{a 1}+C_{b 1}\right) \cos \left(\omega_{g}^{d}|\tau|\right)+\frac{\zeta_{g} \omega_{g}}{\omega_{g}^{d}}\left(C_{a 1}-C_{b 1}\right) \sin \left(\omega_{g}^{d}|\tau|\right)\right) \\
& +\frac{\pi S_{0}}{2 \zeta_{f} \omega_{f}} e^{-\zeta_{f} \omega_{f}|\tau|}\left(\left(C_{a 2}+C_{b 2}\right) \cos \left(\omega_{f}^{d}|\tau|\right)+\frac{\zeta_{f} \omega_{f}}{\omega_{f}^{d}}\left(C_{a 2}-C_{b 2}\right) \sin \left(\omega_{f}^{d}|\tau|\right)\right)
\end{aligned}
$$

where the constants $\omega_{g}^{d}, \omega_{f}^{d}, C_{a 1}, C_{a 2}, C_{b 1}$ and $C_{b 2}$ are defined as indicated below:

$$
\begin{aligned}
\omega_{g}^{d} & =\sqrt{1-\zeta_{g}^{2}} \omega_{g} \\
\omega_{f}^{d} & =\sqrt{1-\zeta_{f}^{2}} \omega_{f} \\
C_{a 1} & =-\frac{\omega_{g}^{6}}{D}\left(\left(1+8 \zeta_{g}^{2}-16 \zeta_{g}^{4}\right)\left(1-\frac{\omega_{g}^{4}}{\omega_{f}^{4}}\right)-8 \zeta_{g}^{2} \frac{\omega_{g}^{2}}{\omega_{f}^{2}}\left(1-2 \zeta_{f}^{2}-\frac{\omega_{g}^{2}}{\omega_{f}^{2}}+2 \zeta_{g}^{2} \frac{\omega_{g}^{2}}{\omega_{f}^{2}}\right)\right) \\
C_{b 1} & =\frac{2 \omega_{g}^{6}}{D}\left(\left(1+8 \zeta_{g}^{2}-16 \zeta_{g}^{4}\right)\left(1-2 \zeta_{g}^{2}-\frac{\omega_{g}^{2}}{\omega_{f}^{2}}+2 \zeta_{f}^{2} \frac{\omega_{g}^{2}}{\omega_{f}^{2}}\right)-2 \zeta_{g}^{2}\left(1-\frac{\omega_{g}^{4}}{\omega_{f}^{4}}\right)\right) \\
C_{a 2} & =\frac{\omega_{g}^{4} \omega_{f}^{2}}{D}\left(\left(1+8 \zeta_{g}^{2}-16 \zeta_{g}^{4}\right)\left(1-\frac{\omega_{g}^{4}}{\omega_{f}^{4}}\right)-8 \zeta_{g}^{2} \frac{\omega_{g}^{2}}{\omega_{f}^{2}}\left(1-2 \zeta_{f}^{2}-\frac{\omega_{g}^{2}}{\omega_{f}^{2}}+2 \zeta_{g}^{2} \frac{\omega_{g}^{2}}{\omega_{f}^{2}}\right)\right) \\
C_{b 2} & =\frac{2 \omega_{g}^{2} \omega_{f}^{4}}{D}\left(\frac{\omega_{g}^{2}}{\omega_{f}^{2}}\left(-\frac{\omega_{g}^{2}}{\omega_{f}^{2}}-8 \zeta_{g}^{2}+16 \zeta_{g}^{2} \zeta_{f}^{2}\right)\left(1-2 \zeta_{g}^{2}-\frac{\omega_{g}^{2}}{\omega_{f}^{2}}+2 \zeta_{f}^{2} \frac{\omega_{g}^{2}}{\omega_{f}^{2}}\right)+2 \zeta_{g}^{2}\left(1-\frac{\omega_{g}^{4}}{\omega_{f}^{4}}\right)\right) \\
D & =-4 \omega_{g}^{2} \omega_{f}^{2}\left(1-2 \zeta_{g}^{2}-\frac{\omega_{g}^{2}}{\omega_{f}^{2}}+2 \zeta_{f}^{2} \frac{\omega_{g}^{2}}{\omega_{f}^{2}}\right)\left(1-2 \zeta_{f}^{2}-\frac{\omega_{g}^{2}}{\omega_{f}^{2}}+2 \zeta_{g}^{2} \frac{\omega_{g}^{2}}{\omega_{f}^{2}}\right)+\omega_{f}^{4}\left(1-\frac{\omega_{g}^{4}}{\omega_{f}^{4}}\right)
\end{aligned}
$$

The above discussion assumes that the ground acceleration can be modeled as a wide-sense stationary 582 stochastic process. It is clear that this is a simplifying assumption, as ground acceleration exhibits a non 583 stationary behavior. A possible means for including such effect in the Clough-Penzien model consists 584 of modulating the white noise bedrock process by means of a deterministic function of time $m(t)$ (see, 585 e.g. [33]). Here, the so-called Shinozuka and Sato modulating function [2] is considered:

$$
m(t)=\frac{1}{c_{3}}\left(e^{-c_{1} t}-e^{-c_{2} t}\right)
$$

where $c_{1}$ and $c_{2}$ are parameters of the model and $c_{3}$ is defined such that the maximum value of the 587 modulating function is equal to unity, yielding:

$$
c_{3}=\frac{c_{1}}{c_{2}-c_{1}} e^{\frac{c_{2}}{c_{2}-c_{1}} \ln \left(\frac{c_{2}}{c_{1}}\right)}
$$


[1] G. Deodatis, Non-stationary stochastic vector processes: seismic ground motion applications, 590 Probabilistic Engineering Mechanics 11 (3) (1996) 149 - 167. doi:https://doi.org/10.1016/0266- 591 $\begin{array}{ll}8920(96) 00007-0 . & 592\end{array}$ URL http://www.sciencedirect.com/science/article/pii/0266892096000070

[2] M. Shinozuka, Y. Sato, Simulation of nonstationary random process, Journal of the Engineering 594 Mechanics Division 93 (1) (1967) 11-40.

[3] M. Beer, Y. Zhang, S. T. Quek, K. K. Phoon, Reliability analysis with scarce information: Com- ${ }^{596}$ paring alternative approaches in a geotechnical engineering context, Structural Safety 41 (2013) 597 1-10. doi:10.1016/j.strusafe.2012.10.003. 598 URL http://dx.doi.org/10.1016/j.strusafe.2012.10.003

[4] M. Beer, S. Ferson, V. Kreinovich, Imprecise probabilities in engineering 600 analyses, Mechanical Systems and Signal Processing $37 \quad(1-2) \quad(2013) \quad 4-29 . \quad 601$ doi:http://dx.doi.org/10.1016/j.ymssp.2013.01.024.

[5] D. Moens, D. Vandepitte, An interval finite element approach for the calculation of envelope 604 frequency response functions, International Journal for Numerical Methods in Engineering 61 (14) 605 (2004) 2480-2507. doi:10.1002/nme.1159.

[6] M. Faes, G. D. Sabyasachi, D. Moens, Hybrid spatial uncertainty analysis for the estima- 608 tion of imprecise failure probabilities in laser sintered pa-12 parts, Computers \& Mathe- 609 matics with Applications 78 (7) (2019) 2395 - 2406, simulation for Additive Manufacturing. 610 doi:https://doi.org/10.1016/j.camwa.2018.08.056. 611 URL http://www.sciencedirect.com/science/article/pii/S0898122118304826 612

[7] W. Gao, D. Wu, C. Song, F. Tin-Loi, X. Li, Hybrid probabilistic interval analysis of bar structures ${ }^{613}$ with uncertainty using a mixed perturbation monte-carlo method, Finite Elements in Analysis and 614 Design 47 (7) (2011) 643-652. 
[8] B. Xia, D. Yu, J. Liu, Hybrid uncertain analysis for structural-acoustic problem with ran- 616 dom and interval parameters, Journal of Sound and Vibration 332 (11) (2013) 2701-2720. 617

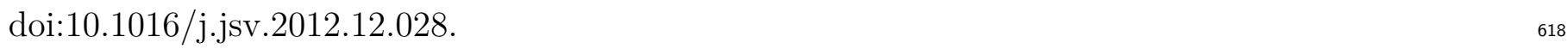

[9] S. Yin, D. Yu, Z. Luo, B. Xia, Unified polynomial expansion for interval and random 619 response analysis of uncertain structure-acoustic system with arbitrary probability distribu- 620 tion, Computer Methods in Applied Mechanics and Engineering 336 (2018) 260 - 285.621 doi:https://doi.org/10.1016/j.cma.2018.03.014.

[10] M. Faes, D. Moens, Recent Trends in the Modeling and Quantification of Non-probabilistic Un- ${ }^{624}$ certainty, Archives of Computational Methods in Engineering (feb 2019). doi:10.1007/s11831-019- ${ }_{625}$ 09327-x.

URL https://doi.org/10.1007/s11831-019-09327-x

[11] J. Wu, Z. Luo, Y. Zhang, N. Zhang, L. Chen, Interval uncertain method for multibody mechanical ${ }_{628}$ systems using Chebyshev inclusion functions, International Journal for Numerical Methods in ${ }_{629}$ Engineering 95 (7) (2013) 608-630. doi:10.1002/nme.4525.

[12] R. Schöbi, B. Sudret, Structural reliability analysis for p-boxes using multi- 631 level meta-models, Probabilistic Engineering Mechanics $48 \quad(2017) \quad 27-38 . \quad 632$ doi:https://doi.org/10.1016/j.probengmech.2017.04.001. URL http://www.sciencedirect.com/science/article/pii/S0266892017300152

[13] R. Schöbi, B. Sudret, Global sensitivity analysis in the context of imprecise probabilities (p-boxes) ${ }^{635}$ using sparse polynomial chaos expansions, Reliability Engineering \& System Safety 187 (2019) 129636 - 141, sensitivity Analysis of Model Output. doi:https://doi.org/10.1016/j.ress.2018.11.021. ${ }_{637}^{637}$ URL http://www.sciencedirect.com/science/article/pii/S0951832017306099 Estimation of Small Failure Probabilities for Strong Nonlinear Models, ASCE-ASME J Risk and ${ }_{640}$ Uncert in Engrg Sys Part B Mech Engrg 5 (4) (dec 2019). doi:10.1115/1.4044044. URL https://asmedigitalcollection.asme.org/risk/article/doi/10.1115/1.4044044/955249/0n-t

[15] J. Sadeghi, M. de Angelis, E. Patelli, Robust propagation of probability boxes by interval predictor ${ }^{643}$ 
models, Structural Safety 82 (2020) 101889. doi:https://doi.org/10.1016/j.strusafe.2019.101889. 644 URL http://www.sciencedirect.com/science/article/pii/S0167473018303035

[16] P. Wei, J. Song, S. Bi, M. Broggi, M. Beer, Z. Lu, Z. Yue, Non-intrusive stochastic analysis with 646 parameterized imprecise probability models: I. performance estimation, Mechanical Systems and 647 Signal Processing 124 (2019) 349 - 368. doi:https://doi.org/10.1016/j.ymssp.2019.01.058. 648 URL http://www.sciencedirect.com/science/article/pii/S0888327019300743

[17] P. Wei, J. Song, S. Bi, M. Broggi, M. Beer, Z. Lu, Z. Yue, Non-intrusive stochastic analysis with 650 parameterized imprecise probability models: II. reliability and rare events analysis, Mechanical Sys- 651 tems and Signal Processing 126 (2019) 227 - 247. doi:https://doi.org/10.1016/j.ymssp.2019.02.015. 652 URL http://www.sciencedirect.com/science/article/pii/S0888327019300986

[18] J. Hurtado, D. Alvarez, J. Ramirez, Fuzzy structural analysis based on fundamental reliability 654 concepts, Computers \& Structures 112-113 (2012) 183-192. doi:10.1016/j.compstruc.2012.08.004. ${ }^{655}$

[19] M. Troffaes, Imprecise monte carlo simulation and iterative importance sampling for the esti- ${ }^{656}$ mation of lower previsions, International Journal of Approximate Reasoning 101 (2018) 31 - 48.657 doi:https://doi.org/10.1016/j.ijar.2018.06.009. 658 URL http://www.sciencedirect.com/science/article/pii/S0888613X17305868

[20] W. Gao, D. Wu, K. Gao, X. Chen, F. Tin-Loi, Structural reliability analysis with 660 imprecise random and interval fields, Applied Mathematical Modelling 55 (2018) 49-67. 661 doi:https://doi.org/10.1016/j.apm.2017.10.029.

URL https://www.sciencedirect.com/science/article/pii/S0307904X17306467

[21] M. Dannert, A. Fau, R. Fleury, M. Broggi, U. Nackenhorst, M. Beer, A probability- ${ }_{664}$ box approach on uncertain correlation lengths by stochastic finite element method, ${ }^{665}$ PAMM (Proceedings in Applied Mathematics and Mechanics) 18 (1) (2018) 666 e201800114. arXiv:https://onlinelibrary.wiley.com/doi/pdf/10.1002/pamm.201800114, $\quad{ }_{667}$ doi:10.1002/pamm.201800114.

[22] M. Faes, D. Moens, Imprecise random field analysis with parametrized kernel functions, Mechanical 670 Systems and Signal Processing 134 (2019) 106334. doi:10.1016/j.ymssp.2019.106334. 
[23] L. Comerford, H. Jensen, F. Mayorga, M. Beer, I. Kougioumtzoglou, Compressive sensing with an 672 adaptive wavelet basis for structural system response and reliability analysis under missing data, 673 Computers \& Structures 182 (2017) 26-40. doi:10.1016/j.compstruc.2016.11.012. 674

URL //www.sciencedirect.com/science/article/pii/S0045794916304618

[24] S. Montoya-Noguera, T. Zhao, Y. Hu, Y. Wang, K.-K. Phoon, Simulation of non- 676 stationary non-gaussian random fields from sparse measurements using bayesian compres- 677 sive sampling and Karhunen-Loève expansion, Structural Safety 79 (2019) $66 \quad$ - $79 . \quad 678$ doi:https://doi.org/10.1016/j.strusafe.2019.03.006. 679 URL http://www.sciencedirect.com/science/article/pii/S0167473018303266 680

[25] Y. Zhang, L. Comerford, I. A. Kougioumtzoglou, E. Patelli, M. Beer, Uncertainty Quantification of ${ }_{681}$ Power Spectrum and Spectral Moments Estimates Subject to Missing Data, ASCE-ASME Journal ${ }_{682}$ of Risk and Uncertainty in Engineering Systems, Part A: Civil Engineering 3 (4) (2017) 04017020.683 doi:10.1061/ajrua6.0000925.

[26] M. Misraji, M. Valdebenito, H. Jensen, C. Mayorga, Application of directional importance 685 sampling for estimation of first excursion probabilities of linear structural systems subject to 686 stochastic Gaussian loading, Mechanical Systems and Signal Processing 139 (2020) 106621. 687 doi:https://doi.org/10.1016/j.ymssp.2020.106621.

[27] A. Chopra, Dynamics of structures: theory and applications to earthquake engineering, Prentice 690 Hall, 1995.

[28] A. Giaralis, P. Spanos, Derivation of response spectrum compatible non-stationary stochastic pro- ${ }^{692}$ cesses relying on Monte Carlo-based peak factor estimation, Earthquakes and Structures 3 (3) 693 (2012) 581-609.

[29] G. Housner, Characteristics of strong-motion earthquakes, Bulletin of the Seismological Society of 695 America 37 (1) (1947) 19-31, cited By 141.

[30] J. Hurtado, Modelación estocástica de la acción sísmica, no. Monografía CIMNE IS-33, Centro ${ }_{697}$ Internacional de Métodos Numéricos en Ingeniería, 1999. 
[31] V. Papadopoulos, D. Giovanis, Stochastic Finite Element Methods, Springer, Cham, 2018.699 doi:10.1007/978-3-319-64528-5.

[32] T. Soong, M. Grigoriu, Random Vibration of Mechanical and Structural Systems, Prentice Hall, 701 Englewood Cliffs, New Jersey, 1993.

[33] C.-H. Yeh, Y. Wen, Modeling of nonstationary ground motion and analysis of inelastic structural 703 response, Structural Safety 8 (1-4) (1990) 281-298.

[34] J. Li, J. Chen, Stochastic Dynamics of Structures, John Wiley \& Sons, Singapore, 2009.705 doi:10.1002/9780470824269.

[35] C. Schenk, G. Schuëller, Uncertainty Assessment of Large Finite Element Systems, Springer-Verlag, 707 Berlin/Heidelberg/New York, 2005.

[36] G. Stefanou, The stochastic finite element method: Past, present and future, Computer Methods 709 in Applied Mechanics and Engineering 198 (9-12) (2009) 1031-1051.

[37] J. Lee, M. Verleysen, Nonlinear Dimensionality Reduction, Springer, 2007.

711

[38] W. Betz, I. Papaioannou, D. Straub, Numerical methods for the discretization of random fields by 712 means of the Karhunen-Loève expansion, Computer Methods in Applied Mechanics and Engineer- 713 ing 271 (2014) 109-129. doi:10.1016/j.cma.2013.12.010. URL http://dx.doi.org/10.1016/j.cma.2013.12.010

[39] W. Gautschi, Numerical Analysis, 2nd Edition, Birkhäuser Boston, 2012. doi:10.1007/978-0-8176- ${ }^{716}$ 8259-0.

[40] S. Au, J. Beck, Estimation of small failure probabilities in high dimensions by subset simulation, 718 Probabilistic Engineering Mechanics 16 (4) (2001) 263-277.

[41] K. Marti, Differentiation of probability functions: The transformation method, Computers \& Math- 720 ematics with Applications 30 (3-6) (1995) 361-382.

[42] Y. Zhang, A. Der Kiureghian, First-excursion probability of uncertain structures, Probabilistic ${ }_{722}$ Engineering Mechanics 9 (1-2) (1994) 135-143.

[43] K. Marti, Approximation and derivatives of probabilities of survival in structural analysis and 724 design, Structural Optimization 13 (4) (1997) 230-243. 
[44] A. A. Taflanidis, J. Beck, Analytical approximation for stationary reliability of certain and uncer- 726 tain linear dynamic systems with higher-dimensional output, Earthquake Engineering \& Structural $\quad 727$ Dynamics 35 (10) (2006) 1247-1267. doi:10.1002/eqe.581.

[45] G. Schuëller, H. Pradlwarter, Benchmark study on reliability estimation in higher dimensions of 729 structural systems - An overview, Structural Safety 29 (2007) 167-182.

[46] S.-S. Lai, Statistical characterization of strong ground motions using power spectral density func- 731 tion, Bulletin of the Seismological Society of America 72 (1) (1982) 259-274.

[47] L. G. Crespo, D. P. Giesy, S. P. Kenny, Interval predictor models with a formal characterization 733 of uncertainty and reliability, Proceedings of the IEEE Conference on Decision and Control 2015- ${ }^{734}$ Febru (February) (2014) 5991-5996. doi:10.1109/CDC.2014.7040327.

[48] M. Imholz, M. Faes, D. Vandepitte, D. Moens, Robust uncertainty quantification in structural 736 dynamics under scarse experimental modal data: A bayesian-interval approach, Journal of Sound 737 and Vibration 467 (2020) 114983. doi:https://doi.org/10.1016/j.jsv.2019.114983.

URL http://www. sciencedirect.com/science/article/pii/S0022460X19305450

[49] M. Faes, J. Sadeghi, M. Broggi, M. de Angelis, E. Patelli, M. Beer, D. Moens, On the Robust ${ }_{740}$ Estimation of Small Failure Probabilities for Strong Nonlinear Models, ASCE-ASME J Risk and 741 Uncert in Engrg Sys Part B Mech Engrg 5 (4) (dec 2019). doi:10.1115/1.4044044.

[50] O. Ditlevsen, R. Olesen, G. Mohr, Solution of a class of load combination problems by directional 743 simulation, Structural Safety 4 (2) (1987) 95-109.

[51] P. Bjerager, Probability integration by directional simulation, Journal of Engineering Mechanics 745 114 (8) (1988) 1285-1297.

[52] O. Ditlevsen, P. Bjerager, R. Olesen, A. Hasofer, Directional simulation in Gaussian processes, 747 Probabilistic Engineering Mechanics 3 (4) (1988) 207 - 217. doi:https://doi.org/10.1016/0266- 748 8920(88)90013-6.

[53] A. Ang, W. Tang, Probability Concepts in Engineering: Emphasis on Applications to Civil and 751 Environmental Engineering, Wiley, 2007. 
[54] A. Der Kiureghian, The geometry of random vibrations and solutions by FORM and SORM, 754 Probabilistic Engineering Mechanics 15 (1) (2000) 81-90.

[55] S. Au, J. Beck, First excursion probabilities for linear systems by very efficient importance sampling, 756 Probabilistic Engineering Mechanics 16 (3) (2001) 193-207.

[56] G. Schuëller, R. Stix, A critical appraisal of methods to determine failure probabilities, Structural 758 Safety 4 (4) (1987) 293-309.

[57] L. Katafygiotis, S. Cheung, Domain decomposition method for calculating the failure probability of 760 linear dynamic systems subjected to Gaussian stochastic loads, Journal of Engineering Mechanics 761 132 (5) (2006) 475-486.

[58] J. Tropp, Topics in sparse approximation, Ph.D. thesis, The University of Texas at Austin (2004). 763

[59] E. Patelli, H. Panayirci, M. Broggi, B. Goller, P. Beaurepaire, H. Pradlwarter, G. Schuëller, Gen- ${ }^{764}$ eral purpose software for efficient uncertainty management of large finite element models, Finite 765 Elements in Analysis and Design 51 (2012) 31-48. doi:10.1016/j.finel.2011.11.003.

[60] A. Zerva, Spatial Variation of Seismic Ground Motions - Modeling and Engineering Applications, 767 CRC Press, 2009.

[61] G. Fu, Seismic response statistics of SDOF system to exponentially modulated coloured input: 769 An explicit solution, Earthquake Engineering \& Structural Dynamics 24 (10) (1995) 1355-1370. 770 doi:10.1002/eqe.4290241006. URL https://onlinelibrary.wiley.com/doi/abs/10.1002/eqe.4290241006 